\title{
C-H activation: a Critical Evaluation of a Published Method and its Application Towards Inherently Chiral Calix[4]arenes
}

\author{
Kevin J. Visagie ${ }^{(\mathbb{D})}$, Luke Hodson ${ }^{\mathbb{D}}$ and Gareth E. Arnott ${ }^{*}$ (ID \\ Department of Chemistry and Polymer Science, Stellenbosch University, Private Bag X1, Matieland, 7602, South Africa.
}

Received 12 September 2019, revised 14 November 2019, accepted 10 January 2020.

\begin{abstract}
C-H activation offers an intriguing access into inherently chiral calix[4]arenes, but has been little explored in the literature. In this article, we report our investigation into a published $\mathrm{C}-\mathrm{H}$ activation method that uses carbamates to direct a palladium catalyzed $\mathrm{C}-\mathrm{H}$ activation and subsequent reaction with $\mathrm{N}$-bromosuccinimide. However, we show that this report is unfortunately flawed on a number of points. An earlier reported study revealed the more likely $S_{E} A r$ mechanism of the bromination reaction, which did not involve palladium catalysis. We nevertheless employed the $S_{E}$ Ar bromination in an attempt to form inherently chiral calix[4]arenes, using a chiral (+)-menthyl carbamate as a directing group. Unfortunately, although the reaction was high yielding, the diastereomers formed were inseparable and we were unable to quantify their ratio. Subsequent removal of the chiral (+)-menthyl carbamate, returned a small positive optical rotation, suggesting that at least a level of asymmetric induction was achieved in the bromination to afford a non-racemic product.
\end{abstract}

KEYWORDS

C-H activation, calix[4]arene, inherent chirality, bromination, diastereoselectivity.

\section{Introduction}

Chirality is and will always be one of the most important aspects of chemistry since all living forms are chiral. From its initial introduction to chemistry students in the form of point chirality (tetrahedral carbon atoms), students later learn that chiral molecules can be formed in many other ways. Our area of research has been focused on one of these aspects, that of inherently chiral calix[4]arenes. ${ }^{1,2}$ Inherently chiral calix[4]arenes can actually be formed by a number of different ways, which makes them attractive targets to study (see Fig. 1 for some simple examples). We have focused on meta-functionalization as a preferred strategy, owing to its similarity to planar chiral ferrocenes, which have a good history of acting as asymmetric ligands. ${ }^{3}$ To date, we have developed some strategies that stereoselectively synthesize meta-functionalized inherently chiral calix[4]arenes using ortho-lithiation chemistry directed by either chiral oxazolines ${ }^{4-6}$ or a sulfoxide. ${ }^{7}$ Whilst these methods are currently the only meaningfully stereoselective methods available to form inherently chiral calix[4]arenes, they suffer from rather difficult chemistry that makes scale-up problematic. To this end, we have been looking at other methods that might generate meta-functionalized inherently chiral calix[4]arenes. One such method involving a putative $\mathrm{C}-\mathrm{H}$ activation pathway caught our attention in the literature. In this 2016 paper by Moghaddam and coworkers, it was reported that methyl carbamates were excellent directing groups for ortho-aryl $\mathrm{C}-\mathrm{H}$ activation (Scheme 1). ${ }^{8}$ We wondered whether the same method could be used on a calix[4]arene to generate inherently chiral versions if the carbamate itself was chiral. Herein we would like to report our preliminary findings in this area, as well as reveal our deep concerns regarding the paper by Moghaddam and co-workers.

* To whom correspondence should be addressed.

E-mail: arnott@sun.ac.za

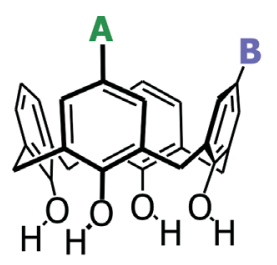

upper-rim

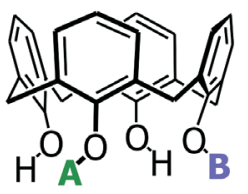

lower-rim

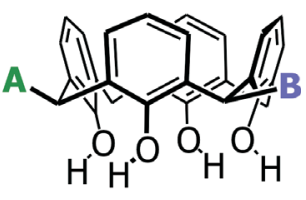

bridges

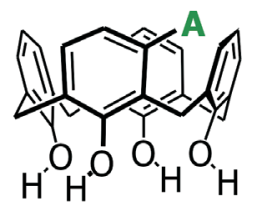

meta
Figure 1 Strategies for creating inherently chiral calix[4]arenes. ${ }^{9}$<smiles>[R]c1cccc(NC(=O)OC)c1</smiles>

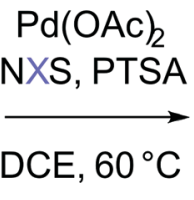<smiles>[R]c1cccc(NC(=O)OC)c1[X]</smiles>

Scheme 1

C-H activation route reported by Moghaddam. ${ }^{8}$

\section{Results and Discussion}

\subsection{Model Study}

Our investigation began with a model study, primarily to ascertain whether the chemistry reported by Moghaddam and 
co-workers was itself correct (Scheme 1). We had some initial concerns about the reported method, as the paper appeared to have superficial errors that we found surprising. Some of these errors might have been due to topographical oversight, but did warrant further investigation. One concern was that the reported method should have been theoretically possible using just $N$-bromosuccinimide (NBS), without any need for the palladium catalyst. In the paper, the authors reported that the reaction failed using NBS in acetonitrile, but only worked when palladium acetate was added. The authors then reported that the reaction failed in dichloroethane (DCE) even with palladium acetate, but worked again when para-toluenesulfonic acid (PTSA) was added. The published table of results showed no example of an experiment that then excluded the palladium catalyst, but kept PTSA, i.e. a control experiment. However, the text did report that a control experiment had been performed and then referred to an incorrect entry on the table (hence a possible typographical error). For this reason, we decided to have a closer look at the reaction ourselves.

The model selected (carbamate 1) included a para-methoxy group, which served both as a model for a single functionalised aromatic ring on the calix[4]arene, and as a means for testing the directing ability of the carbamate vs. the methoxy group. Essentially, it was found that the role of the palladium in this experiment was greatly exaggerated (see Table 1), with the yield of brominated 2 only being marginally higher when it was included. In both cases, the carbamate was the sole director towards ortho-bromination. It therefore seems likely to us that Moghaddam and co-workers had somewhat overstated the importance of the palladium and its role in the reaction.

With this rather unsurprising result, we took a much closer look at the paper by Moghaddam and co-workers and noticed more problems. In the introduction, they made the main claim that: 'To the best of our knowledge, this is the first report of
Table 1 Model study to examine the role of the palladium catalyst.<smiles>COC(=O)Nc1ccc(OC)cc1</smiles>

1

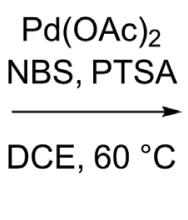<smiles>COC(=O)Nc1ccc(OC)cc1Br</smiles>

2

\begin{tabular}{ccccc}
\hline Entry & $\operatorname{Pd}(\mathrm{OAc})_{2}$ & PTSA $^{\mathrm{a}}$ & Time & Yield \\
\hline $\mathbf{1}$ & $5 \mathrm{~mol} \%$ & $50 \mathrm{~mol} \%$ & $2.5 \mathrm{~h}$ & $79 \%$ \\
$\mathbf{2}$ & - & $50 \mathrm{~mol} \%$ & $2.5 \mathrm{~h}$ & $69 \%$ \\
\hline
\end{tabular}

a The acid is important in this reaction; without it the rate drops dramatically

application of $\mathrm{N}$-arylcarbamates as DG in C-halogen bond formation'. This statement cannot be proven false, since it is 'to the best of their knowledge', but it is nevertheless wrong. A quick search on Reaxsys reveals a different story: excluding papers reported after their own 2016 publication, 43 documents (including patents) report the use of NBS brominating an aryl ring ortho to a carbamate; 12 documents using NCS (chlorination) and 24 documents using NIS (iodination). Many further examples can also be found employing the respective molecular halogen reagent (e.g. chlorine, bromine and iodine). A minor selection of examples from the peer review literature are shown in Fig. 2 (refer to Supplementary Information). It is disheartening that the reviewers never noticed this, since this fact alone puts a completely different interpretation onto the results presented.

Secondly, Moghaddam and co-workers cite a 2014 paper by<smiles>CCCOC(=O)Nc1cc(OCc2ccccc2)c2ccccc2c1</smiles>

NBS

$\underset{\mathrm{THF},-60^{\circ} \mathrm{C}}{\stackrel{\mathrm{H}_{2} \mathrm{SO}_{4} \text { (cat) }}{\longrightarrow}}$

$5 \mathrm{~h}$

Boger et al. JACS 1989<smiles>CCCCOC(=O)Nc1cc2c3c(cc(C(C)CC)nc3c1NC(=O)OCCC)C(=O)N2</smiles><smiles>CCCCOC(=O)Nc1cc(OCCCC)c2ccccc2c1Br</smiles><smiles>COC(=O)Nc1ccccc1C</smiles>
SynthCommun 2009<smiles>COC(=O)Nc1ccc(Cl)cc1Cl</smiles><smiles>CCCCC(=O)OC(=O)Nc1c(Cl)c(NC(=O)OCCC)c2nc(C(=O)OCC)cc3c2c1NC3=O</smiles><smiles>COC(=O)c1ccc(N2C(=O)OCC2C)cc1OC</smiles>

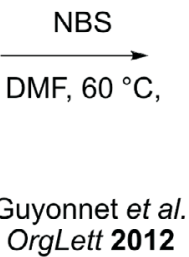<smiles>COC(=O)c1cc(Br)c(N2C(=O)OCC2C)cc1OC</smiles>

OrgLett 2010<smiles>COC(=O)Nc1ccc(Br)c(C)c1</smiles><smiles>COC(=O)Nc1cc(C)c(Br)cc1I</smiles><smiles>CCOC(=O)Nc1ccc2cc(F)ccc2c1Cl</smiles>

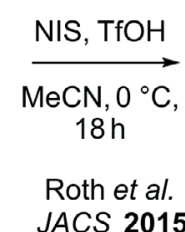

Figure 2 Selected reports ${ }^{10-15}$ using NXS reagents and N-arylcarbamates prior to Moghaddam and co-workers' paper. ${ }^{*}=$ not disclosed. 
Uhlig and $\mathrm{Li}^{16}$ making the following statement: 'Although they are structurally and electronically similar to O-aryl carbamates, after being introduced by $\mathrm{Li}$ et al. as an effective and removable DG, N-arylcarbamates have not been investigated as $\mathrm{C}-\mathrm{H}$ activation DG(sic).' This statement is false, since Uhlig and $\mathrm{Li}$ very definitely reported on $\mathrm{N}$-arylcarbamates being used as $\mathrm{C}-\mathrm{H}$ activation directing groups. In fact, it is the entire focus of their paper, which is titled 'Aniline Carbamates: A Versatile and Removable Motif for Palladium-Catalyzed Directed C-H Activation'. In Uhlig and Li's paper, they also had a good look at the reaction mechanism and found that the aniline carbamate strongly favoured the promotion of electrophilic aromatic substitution, which aligns with the observations in the literature that NXS is itself capable of halogenating ortho to an $\mathrm{N}$-arylcarbamate via a non-C-H activation pathway.

Thirdly, closer inspection of Moghaddam and co-workers' proposed mechanism also reveals a number of problems. Firstly, an intermediate involving a deprotonated carbamate is proposed, which is unlikely since the reaction is under acidic conditions, and secondly, they make no account of the purpose of the $p$-toluene sulfonic acid which they point out is crucial; see ref. $^{8}$ : Scheme 5. A more realistic and plausible mechanism can be found in the paper by Uhlig and Li, which they cited. ${ }^{16}$ Overall, it is our opinion that Moghaddam and co-workers submitted a paper that failed to acknowledge the correct state of the art, and in so doing failed to understand the more likely interpretation of their results. The work by Uhlig and Li makes it clear that $\mathrm{C}-\mathrm{H}$ activation is almost certainly occurring, but in the case of halogenation, this may not be the main mechanistic pathway. The unfortunate thing is that this paper has been cited many times, including four reviews where its conclusions have been reported without question..$^{17-20}$

\subsection{Calix[4]arene Study}

Whilst our starting point for the study proved to be somewhat spurious, the use of a chiral carbamate to potentially form inherently chiral calix[4]arenes was deemed to be worth pursuing. First an achiral model study was carried out, in order to check the chemistry, by reacting the known mono-aminocalix[4]arene $3^{21,22}$ with methyl chloroformate and pyridine (Scheme 2). The carbamate product 4 was confirmed via ${ }^{1} \mathrm{H} N M R$ spectroscopy (singlet at $\delta 3.69 \mathrm{ppm}$ for the hydrogen atoms of the methoxy group), infrared $\left(1727 \mathrm{~cm}^{-1} \text { for the carbamate }\right)^{23}$ and HRMS (calculated for $\mathrm{C}_{42} \mathrm{H}_{52} \mathrm{NO}_{6}[\mathrm{M}+\mathrm{H}]^{+}$: 666.3790; found 666.3782). With this material in hand, we attempted the bromination using both Moghaddam and co-workers' method, against one that excluded the palladium catalyst. Once again, the yields of the reaction were only marginally higher when the palladium catalyst was included, suggesting that the dominant mechanism was still the electrophilic bromination and not $\mathrm{C}-\mathrm{H}$ activation (see Table 2). What was also worth noting was that the unfuctionalized aromatic rings were not brominated, even

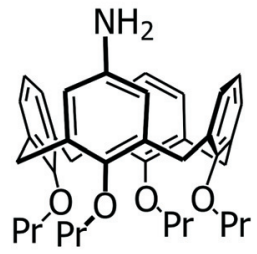

3

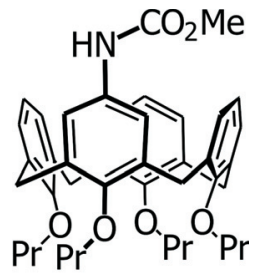

4
Scheme 2

Synthesis of mono-functionalised calix[4]arene carbamate model.
Table 2 Bromination results on mono-functionalized calix[4]arene.

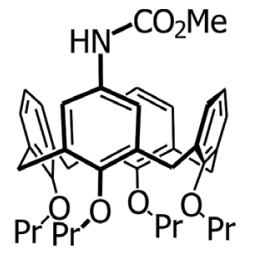

4

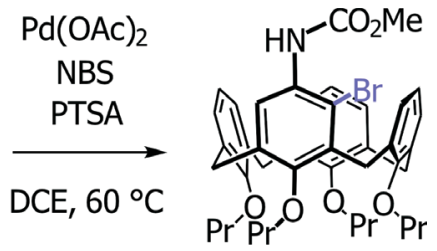

5

\begin{tabular}{ccccc}
\hline Entry & $\operatorname{Pd}(\mathrm{OAc})_{2}$ & PTSA & Time & Yield \\
\hline $\mathbf{1}$ & $5 \mathrm{~mol} \%$ & $50 \mathrm{~mol} \%$ & $2.5 \mathrm{~h}$ & $87 \%$ \\
$\mathbf{2}$ & - & $50 \mathrm{~mol} \%$ & $2.5 \mathrm{~h}$ & $77 \%$ \\
\hline
\end{tabular}

though they are in principle activated by the para-propoxy groups. This is again likely due to the greater directing effect of the carbamate as evidenced in the model study (see earlier). Successful synthesis of the ortho-brominated product 5 was primarily concluded from the HRMS (calculated for $\mathrm{C}_{42} \mathrm{H}_{51} \mathrm{BrNO}_{6}$ $[\mathrm{M}+\mathrm{H}]^{+}:$744.2900; found 744.2872 with matching isotopic distribution), and ${ }^{1} \mathrm{H}$ NMR spectroscopy (whose aromatic region revealed the loss of one proton).

Having established that the carbamate was a suitable functional group for activating the calix[4]arene meta-position, we turned our attention to using a chiral carbamate to see whether diastereoselectivity could be achieved. For this we selected (1S)-(+)-menthyl chloroformate (it was available in our labs), and reacted it with mono-amine calix[4]arene 3 under the same conditions as before. The new chiral carbamate 6 was obtained in excellent yields between 90 and $98 \%$ after work-up and column chromatography. The mono-menthyl carbamate calix[4]arene 6 was characterized by NMR spectroscopy, HRMS (calculated for $\mathrm{C}_{51} \mathrm{H}_{68} \mathrm{NO}_{6}[\mathrm{M}+\mathrm{H}]^{+}:$790.5047; found 790.5040) and infrared (1697 $\mathrm{cm}^{-1}$ for carbamate).

With our chiral carbamate in hand, we attempted a selective bromination reaction using the protocol without palladium, which returned a good yield $(>80 \%)$ for the inseparable brominated products $7 \mathbf{a}$ and $7 \mathbf{b}$. We had been hoping to use ${ }^{1} \mathrm{H}$ NMR spectroscopy to quantify the diastereoselectivity of the reaction, but to our disappointment, there were really no promising signals to work with. Different solvents $\left(\mathrm{CDCl}_{3}\right.$, DMSO- $\mathrm{d}_{6}$ and $\mathrm{C}_{6} \mathrm{H}_{6}$ ) and different temperatures (up to the maximum allowed) all failed to help us determine the diastereoselectivity. The only signal that appeared marginally useful was the methine signal on the chiral centre of the menthyl group. In the starting material this appeared at $\delta 4.60 \mathrm{ppm}$ as a triplet of doublets, whilst in the product it appeared as a multiplet centred around $\delta 4.69$ ppm (see Fig. 3). On close inspection, it could be seen that this multiplet was an overlay of two triplets of doublets in essentially a 1:1 ratio, suggesting a poor diastereoselectivity in the reaction.

In order to see if we could improve on this diastereoselectivity, we tried the reaction at lower temperatures. Since DCE is relatively limited in this sense, we changed the solvent to dichloromethane (DCM) and initiated a temperature study (Table 3). Unfortunately, in all cases (down to $-35^{\circ} \mathrm{C}$ ) we saw no discernible improvement in the diastereoselectivity (as judged by the aforementioned signals in the ${ }^{1} \mathrm{H}$ NMR spectra). However, what was unexpected was just how well the reaction occurred even at lower temperatures, albeit with slightly longer reaction times.

Our inability to determine the ratio of diastereomers in this 
(a) brominated
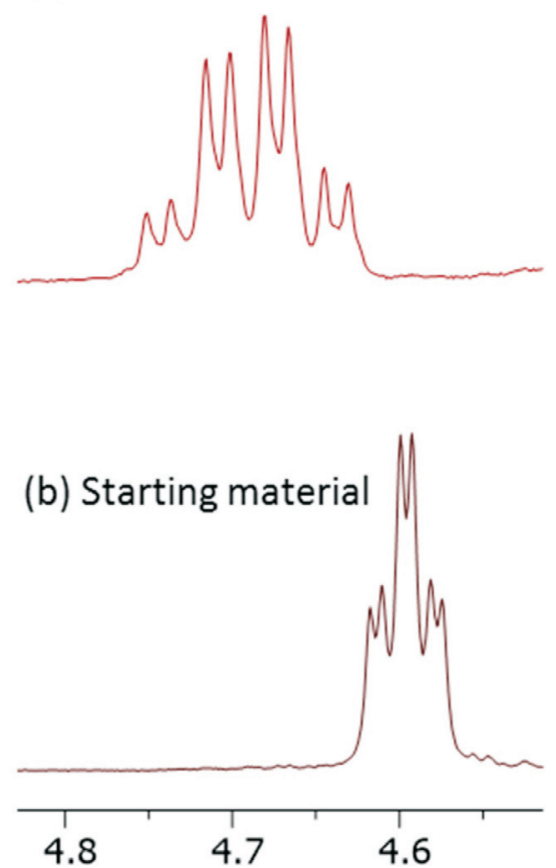

Figure 3 Comparison of the chiral C-H methine in the brominated $7 \mathrm{a}$ and $7 \mathbf{b}(\mathbf{a})$ and starting material $\mathbf{6}(\mathbf{b})$ menthyl carbamate calix[4]arenes (spectra run in $\mathrm{CDCl}_{3}$ ).

Table 3 Results for lower temperature study for bromination of menthylcarbamate calix[4]arene. Reagents and conditions: calix[4]arene 6 (1 equiv), NBS (1.1 equiv), PTSA ( 0.5 equiv), DCM.

\begin{tabular}{ccccc}
\hline Entry & Temp. $/{ }^{\circ} \mathrm{C}$ & Catalyst & Time/h & Yield $/ \%$ \\
\hline 1 & 25 & $\operatorname{Pd}(\mathrm{OAc})_{2}$ & 4 & 90 \\
2 & 25 & - & 4 & 86 \\
3 & 0 & $\mathrm{Pd}(\mathrm{OAc})_{2}$ & 4 & 90 \\
4 & 0 & - & 4 & 82 \\
5 & -35 & $\mathrm{Pd}(\mathrm{OAc})_{2}$ & 4 & 92 \\
6 & -35 & - & 4 & 82 \\
\hline
\end{tabular}

reaction was frustrating. Even normal and reversed-phased HPLC experiments, including the use of a chiral column, failed to separate the two diastereomers. As a last resort, we decided to remove the chiral menthyl group and examine the optical rotation of the resultant mixture of enantiomers to see if any optical activity was displayed. Although this would not be a means of determining enantioselectivity, the optical rotation would at least point to whether any chiral induction had taken place. Removal of the menthyl group was readily achieved using Coudert's method of tetra- $n$-butylammonium fluoride $\left(\mathrm{Bu}_{4} \mathrm{NF}\right.$, TBAF) in THF (Scheme 3). ${ }^{24}$ The reaction, as expected, ${ }^{24}$ was sluggish and required heating under reflux for $36 \mathrm{~h}$. Nevertheless, after work-up and purification, the aminocalix[4]arene product was obtained in yields $>80 \%$. The ${ }^{1} \mathrm{H}$ NMR spectrum showed the complete removal of the menthyl group, greatly simplifying the spectrum. The loss of the carbamate was also detected by IR spectroscopy and the HRMS returned the expected molecular ion (and isotopic distribution pattern) for the product. Optical rotation experiments were then run on material generated from bromination at $-35^{\circ} \mathrm{C}$ (Table 3 , entries 5 and 6), returning values of $[\alpha]_{D}=+6^{\circ}$ and $+3^{\circ}$ for products derived from entries 5 and 6 , respectively. Whilst these values cannot be used for any form of quantification, they do indicate a<smiles>CCCOc1ccccc1OP(OCC)OCc1ccccc1N</smiles><smiles>CC(C)C1CCC(C(C)C)C(C(=O)OC(=O)Cl)C1</smiles>
3

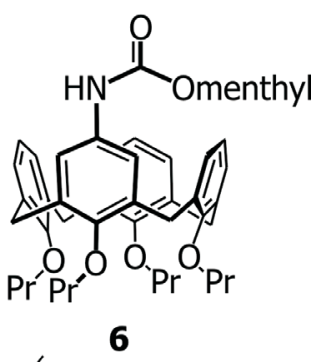

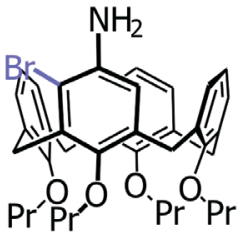

8a

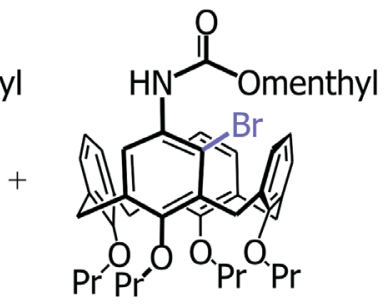

7b

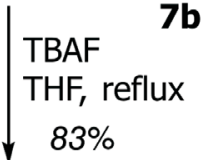<smiles>CCCOc1cccc(P(OCCC)Oc2cccc(OCCC)c2OCCC)c1O</smiles>

8b

Scheme 3

Synthesis of brominated menthyl carbamate calix[4]arene diastereomers.

level of enantiomeric excess, which in turn, points to at least some degree of diastereoselectivity induced by the chiral menthyl carbamate.

\section{Conclusion}

In conclusion, we have shown that a report in the literature claiming a $\mathrm{C}-\mathrm{H}$ activation route, mediated by a catalytic palladium in which a carbamate directs the formation of an aryl halide bond, is somewhat overstated and incorrect on a number of points claimed. Nevertheless, using a chiral calix[4]arene carbamate, bromination successfully delivered a product that suggested a modest level of inherent chirality that could not be quantified. Further work can potentially look at other chiral groups and also at extending the number of directing groups on the upper-rim of the calix[4] arene to two or even four, in order to access more interesting meta-functionalized calix[4]arenes.

\section{Supplementary Material}

Copies of NMR, IR and HRMS spectra for all new compounds synthesized and also references for halogenation reactions prior to 2016 are provided in the supplementary material appended to the end of this article.

\section{Experimental}

All chemicals were purchased from Merck or Sigma-Aldrich. Dichloromethane was dried from calcium hydride under nitro- 
gen. Other reagents that required purification were done so according to standard procedures. The synthesis of methyl (4-methoxyphenyl)carbamate $\mathbf{1}$ was performed using a literature procedure from $p$-anisidine, ${ }^{25}$ and mono-aminocalix[4] arene 3 was prepared as previously reported by us. ${ }^{22}$

For syntheses performed under inert conditions the glassware was oven-dried and then placed under vacuum of $<0.5 \mathrm{~mm} \mathrm{Hg}$ before being periodically flushed with argon until reaching room temperature. All reactions were performed under positive pressure of $2.8 \mathrm{kPa}$ of 5.0 grade argon (Air Products). Low temperature reactions were performed in a Dewar containing ice and acetone $\left(-15^{\circ} \mathrm{C}\right)$, solid $\mathrm{CO}_{2}$ and acetonitrile $\left(-40^{\circ} \mathrm{C}\right)$ or solid $\mathrm{CO}_{2}$ and acetone $\left(-78^{\circ} \mathrm{C}\right)$.

Column chromatography was performed using 230-400 nm silica and thin layer chromatography (TLC) was performed using Macherey-Nagel DC-Fertigfolien ALUGRAM Xtra SIL G/UV254 TLC plates. Visualization of compounds on TLC plates was performed by using a UV lamp or using a cerium ammonium molybdate (CAM) solution followed by heating.

Both ${ }^{1} \mathrm{H}$ and ${ }^{13} \mathrm{C}$ NMR spectra were obtained using Varian 300 $\mathrm{MHz}$ VNMRS, Varian $400 \mathrm{MHz}$ Unity INOVA and Varian 600 $\mathrm{MHz}$ Unity INOVA NMR instruments. Chemical shifts were recorded using the residual solvent peaks (chloroform- $d$ or DMSO- $d_{6}$ ) and reported in ppm. Unless otherwise stated, NMR spectra was obtained at room temperature. All mass spectrometry spectra were obtained by Central Analytical Facility (CAF) at Stellenbosch University using a Waters API Q-TOF Ultima mass spectrometer. IR spectra were obtained using a Thermo Nicolet Nexus FTIR instrument using the ATR attachment. Melting points were obtained using a Gallenkamp Melting Point Apparatus.

\section{Methyl (2-bromo-4-methoxyphenyl)carbamate (2)}

An oven-dried Schlenk equipped with a magnetic stir bar and flushed with argon was charged with 1 (100 mg, $0.552 \mathrm{mmol})$, NBS (108.5 mg, $1.1 \mathrm{eq})$, PTSA (48 mg, $0.5 \mathrm{eq})$ and DCE (1.1 mL). The contents were then heated to $60{ }^{\circ} \mathrm{C}$ and left to stir for $2.5 \mathrm{~h}$. After $2.5 \mathrm{~h}$, the reaction contents were cooled to room temperature and then diluted with DCM $(20 \mathrm{~mL})$. The solution was then poured into $\mathrm{H}_{2} \mathrm{O}(20 \mathrm{~mL})$ after which the product was extracted with $\mathrm{DCM}(10 \mathrm{~mL} \times 3)$. The organic layers were subsequently combined and washed with a $10 \% \mathrm{HCl}$ solution $(20 \mathrm{~mL})$, followed by sat. $\mathrm{NaHCO}_{3}(20 \mathrm{~mL})$ solution and finally brine $(20 \mathrm{~mL})$. The solution was then dried over $\mathrm{MgSO}_{4}$ and the solvent was removed via reduced pressure. Purification was achieved via silica gel flash column chromatography (EtOAc: PET 10:90) to obtain compound 2 as an orange solid in $69 \%$ yield (99 mg).

The characterisation data collected for this compound compared well to literature data. ${ }^{8}$

${ }^{1} \mathrm{H}$ NMR (400 MHz, CHLOROFORM- $d$ ) $\delta$ ppm 7.93 (br. s, $1 \mathrm{H}$, $\mathrm{NH}), 7.07\left(\mathrm{~d},{ }^{4} \mathrm{~J}_{\mathrm{HH}}=2.9 \mathrm{~Hz}, 1 \mathrm{H}, \mathrm{ArH}\right), 6.90-6.82(\mathrm{~m}, 2 \mathrm{H}, \mathrm{ArH}), 3.78$ (s, $\left.3 \mathrm{H}, \mathrm{OCH}_{3}\right) 3.77\left(\mathrm{~s}, 3 \mathrm{H}, \mathrm{OCH}_{3}\right)$.

5-Methyl carbamate-25,26,27,28-tetrapropoxycalix[4]arene (4)

To an oven-dried 2-neck round-bottomed flask, compound 3 (375 mg, $0.62 \mathrm{mmol})$ dissolved in DCM $(20 \mathrm{~mL})$ and pyridine $(74.0 \mu \mathrm{L}, 0.92 \mathrm{mmol}, 1.5 \mathrm{eq})$ was added. After cooling the mixture to $0{ }^{\circ} \mathrm{C}$, methyl chloroformate $(71.5 \mu \mathrm{L}, 0.92 \mathrm{mmol}, 1.5 \mathrm{eq})$ was added. The reaction was then allowed to warm to room temperature and after $30 \mathrm{~min}$ the reaction had run to completion. $\mathrm{H}_{2} \mathrm{O}$ $(20 \mathrm{~mL})$ was added to the reaction mixture and the product was subsequently extracted with DCM $(10 \mathrm{~mL} \times 3)$. The organic layers were combined and first washed with a dilute $\mathrm{HCl}$ solution
$(0.2 \mathrm{M}, 25 \mathrm{~mL})$, brine $(25 \mathrm{~mL})$ and dried over $\mathrm{MgSO}_{4}$ before removing excess solvent under vacuo. The crude product was purified via silica gel flash column chromatography (EtOAc:PET 5:95) to afford compound 4 as a colourless glass (370 $\mathrm{mg}, 90 \%$ ). $\boldsymbol{R}_{f}=0.66$ (10:90 EtOAc:PET); Mp = 124-128 ${ }^{\circ} \mathrm{C}$; IR $\left(\right.$ ATR, $\left.\mathbf{c m}^{-1}\right)$ : $3374(\mathrm{~N}-\mathrm{H}), 2960$ and $2873(\mathrm{C}-\mathrm{H}), 1727(\mathrm{C}=\mathrm{O}), 1529$ (arene), 1454 $(\mathrm{C}=\mathrm{C}), 1211$ and 1191 (C-O-C), 1005 and $966(\mathrm{C}-\mathrm{N}), 757(\mathrm{C}-\mathrm{H})$; ${ }^{1} \mathrm{H}$ NMR (300 MHz, CHLOROFORM- $d$ ) $\delta$ ppm 6.86-6.36 (br m, $11 \mathrm{H}, \mathrm{ArH}), 6.13$ (br s, 1H, NHR), $4.47\left(\mathrm{~d}, 2 \mathrm{H},{ }^{2} \mathrm{~J}_{\mathrm{HH}}=13.4 \mathrm{~Hz}\right.$, $\left.\mathrm{ArCH}_{2(a x) .)} \mathrm{Ar}\right), 4.42\left(\mathrm{~d}, 2 \mathrm{H},{ }^{2} J_{\mathrm{HH}}=13.4 \mathrm{~Hz}, \mathrm{ArCH}_{2(a x .)} \mathrm{Ar}\right), 3.92-3.76$ $\left(\mathrm{m}, 8 \mathrm{H}, \mathrm{OCH}_{2} \mathrm{CH}_{2}\right), 3.70\left(\mathrm{~s}, 3 \mathrm{H}, \mathrm{OCH}_{3}\right), 3.16\left(\mathrm{~d}, 2 \mathrm{H},{ }^{2} J_{\mathrm{HH}}=13.4 \mathrm{~Hz}\right.$, $\left.\mathrm{ArCH}_{2(\text { eq })} \mathrm{Ar}\right), 3.13\left(\mathrm{~d}, 2 \mathrm{H},{ }^{2} J_{\mathrm{HH}}=13.4 \mathrm{~Hz}, \mathrm{ArCH}_{2(\text { eq. })} \mathrm{Ar}\right), 2.00-1.84$ $\left(\mathrm{m}, 8 \mathrm{H}, \mathrm{CH}_{2} \mathrm{CH}_{2} \mathrm{CH}_{3}\right), 1.08-0.94\left(\mathrm{~m}, 12 \mathrm{H}, \mathrm{CH}_{2} \mathrm{CH}_{2} \mathrm{CH}_{3}\right) ;{ }^{13} \mathrm{C}\{\mathbf{1 H}\}$ (75 MHz, CHLOROFORM- $d$ ) $\delta$ ppm 157.0 (ArC), 156.5 (NHCOO), 153.0 (ArC), 135.6 (ArC) 135.5 (ArC), $135.3(\operatorname{ArC}), 135.0$ $(\mathrm{ArC}), 131.5(\operatorname{ArC}), 128.5(\operatorname{ArC}), 128.4(\operatorname{ArC}), 128.1(\operatorname{ArC}), 122.1$ $(\mathrm{ArC}), 121.6(\mathrm{ArC}), 119.4(\mathrm{ArC}), 76.80\left(\mathrm{OCH}_{2} \mathrm{CH}_{2}\right), 76.76$ $\left(\mathrm{OCH}_{2} \mathrm{CH}_{2}\right), 52.2\left(\mathrm{OCH}_{3}\right), 31.2\left(\mathrm{ArCH} \mathrm{H}_{2} \mathrm{Ar}\right), 31.1\left(\mathrm{ArCH}_{2} \mathrm{Ar}\right), 23.43$ $\left(\mathrm{OCH}_{2} \mathrm{CH}_{2} \mathrm{CH}_{3}\right), 23.37\left(\mathrm{OCH}_{2} \mathrm{CH}_{2} \mathrm{CH}_{3}\right), 23.3\left(\mathrm{OCH}_{2} \mathrm{CH}_{2} \mathrm{CH}_{3}\right), 10.6$ $\left(\mathrm{OCH}_{2} \mathrm{CH}_{2} \mathrm{CH}_{3}\right), 10.4\left(\mathrm{OCH}_{2} \mathrm{CH}_{2} \mathrm{CH}_{3}\right)$; HRMS-Positive: $\mathrm{m} / \mathrm{z}$ $[\mathrm{M}+\mathrm{H}]^{+}$calcd. for $\mathrm{C}_{42} \mathrm{H}_{52} \mathrm{NO}_{6}: 666.3795$; found 666.3782.

(士)-6-Bromo-5-methyl carbamate-25,26,27,28-tetrapropoxycalix[4]arene (5)

A Schlenk equipped with a magnetic stir bar and flushed with argon was charged with compound 4 (97 $\mathrm{mg}, 0.15 \mathrm{mmol})$, NBS (28 mg, $0.16 \mathrm{mmol}, 1.1 \mathrm{eq})$, PTSA (14 mg, $0.071 \mathrm{mmol}, 0.5 \mathrm{eq})$ and $\mathrm{Pd}(\mathrm{OAc})_{2}(1.6 \mathrm{mg}, 0.007 \mathrm{mmol}, 0.05 \mathrm{eq})$ in DCE $(2 \mathrm{~mL})$. The contents were heated to $60^{\circ} \mathrm{C}$ and left to stir for two and a half hours. After the allotted time, the reaction was cooled to room temperature and diluted with DCM $(5 \mathrm{~mL})$ before being poured into $\mathrm{H}_{2} \mathrm{O}(10 \mathrm{~mL})$. The product was extracted with DCM $(5 \mathrm{~mL} \times$ $3)$ and the combined organic layers were subsequently washed with $10 \% \mathrm{HCl}(10 \mathrm{~mL})$, sat. $\mathrm{NaHCO}_{3}(10 \mathrm{~mL})$ and finally brine $(10 \mathrm{~mL})$. The solution was then dried over $\mathrm{MgSO}_{4}$ and the solvent was removed via reduced pressure. Purification was achieved via silica gel flash column chromatography (2:98 EtOAc:PET) to yield compound 5 as an amorphous glass $(90 \mathrm{mg}$, $82 \%) . R_{f}=0.70(\mathrm{DCM}) ; \mathbf{M p}=174-184{ }^{\circ} \mathrm{C}$; IR $\left(\right.$ ATR, $\left.\mathbf{c m}^{-1}\right): 2957$ and $2873(\mathrm{C}-\mathrm{H}), 2365(\mathrm{~N}-\mathrm{H}), 1705(\mathrm{C}=\mathrm{O}), 1455(\mathrm{C}=\mathrm{C}), 1192$ and 1087 (C-O-C), 1005 and 965 (C-N), 762 (C-H); ${ }^{1} \mathrm{H}$ NMR (300 MHz, CHLOROFORM- $d$ ) $\delta$ ppm 7.86 (br s, $1 \mathrm{H}, \mathrm{NH}), 7.13$ (br, 1H, ArH), $7.09\left(\mathrm{~d}, 2 \mathrm{H},{ }^{2} J_{H H}=7.4 \mathrm{~Hz}, \operatorname{ArH}\right), 6.90\left(\mathrm{t}, 1 \mathrm{H},{ }^{3} J_{\mathrm{HH}}=7.4 \mathrm{~Hz}, \mathrm{ArH}\right)$ 6.40-6.09 (m, 6H, ArH), 4.49-4.36 (m, 4H, ArCH $\left.{ }_{2(a x .)} \mathrm{Ar}\right), 4.11-3.81$ $\left(\mathrm{m}, 4 \mathrm{H}, \mathrm{OCH}_{2} \mathrm{CH}_{2}\right.$ and $\left.1 \mathrm{H}, \mathrm{ArCH}_{2(e q) .} \mathrm{Ar}\right), 3.83$ (s, $\left.3 \mathrm{H}, \mathrm{OCH}_{3}\right)$, 3.74-3.63 (m, 4H, $\left.\mathrm{OCH}_{2} \mathrm{CH}_{2}\right), 3.27-3.06\left(\mathrm{~m}, 3 \mathrm{H}, \mathrm{ArCH}_{2(\text { eq. })} \mathrm{Ar}\right)$, 2.08-1.79 (m, 8H, $\left.\mathrm{CH}_{2} \mathrm{CH}_{2} \mathrm{CH}_{3}\right), 1.10\left(\mathrm{t},{ }^{3} J_{\mathrm{HH}}=7.4 \mathrm{~Hz}, 6 \mathrm{H}\right.$, $\left.\mathrm{CH}_{2} \mathrm{CH}_{2} \mathrm{CH}_{3}\right), 0.91\left(\mathrm{t},{ }^{3} J_{\mathrm{HH}}=7.4 \mathrm{~Hz}, 3 \mathrm{H}, \mathrm{CH}_{2} \mathrm{CH}_{2} \mathrm{CH}_{3}\right), 0.90\left(\mathrm{t},{ }^{3} J_{\mathrm{HH}}\right.$ $\left.=7.4 \mathrm{~Hz}, 3 \mathrm{H}, \mathrm{CH}_{2} \mathrm{CH}_{2} \mathrm{CH}_{3}\right) ;{ }^{13} \mathrm{C}\{\mathbf{1 H}\}(75 \mathrm{MHz}, \mathrm{CHLORO}-$ FORM- $d$ ) $\delta$ ppm $158.0(\operatorname{ArC}), 155.3(\mathrm{NHCOO}), 154.6(\operatorname{ArC}), 154.3$ $(\operatorname{ArC}), 137.6(\operatorname{ArC}), 137.1(\operatorname{ArC}), 137.0(\operatorname{ArC}), 136.7(\operatorname{ArC}), 135.5$ $(\operatorname{ArC}), 133.5(\operatorname{ArC}), 132.4(\operatorname{ArC}), 132.1(\operatorname{ArC}), 130.2(\operatorname{ArC}), 129.0$ (ArC), $128.9(\operatorname{ArC}), 127.9(\operatorname{ArC}), 127.8(\operatorname{ArC}), 127.5(\operatorname{ArC}), 126.6$ (ArC), 122.3 (ArC), 122.3 (ArC), 121.9 (ArC), 121.0 (ArC), 77.1 $\left(\mathrm{OCH}_{2} \mathrm{CH}_{2}\right), 77.0\left(\mathrm{OCH}_{2} \mathrm{CH}_{2}\right), 76.6\left(\mathrm{OCH}_{2} \mathrm{CH}_{2}\right), 52.6\left(\mathrm{OCH}_{3}\right), 31.2$ $\left(\mathrm{ArCH}_{2} \mathrm{Ar}\right), 31.1\left(\mathrm{ArCH}_{2} \mathrm{Ar}\right), 31.0\left(\mathrm{ArCH}_{2} \mathrm{Ar}\right), 30.2\left(\mathrm{ArCH} \mathrm{H}_{2} \mathrm{Ar}\right), 23.65$ $\left(\mathrm{OCH}_{2} \mathrm{CH}_{2} \mathrm{CH}_{3}\right), 23.59\left(\mathrm{OCH}_{2} \mathrm{CH}_{2} \mathrm{CH}_{3}\right), 23.1\left(\mathrm{OCH}_{2} \mathrm{CH}_{2} \mathrm{CH}_{3}\right), 23.0$ $\left(\mathrm{OCH}_{2} \mathrm{CH}_{2} \mathrm{CH}_{3}\right), 10.94\left(\mathrm{OCH}_{2} \mathrm{CH}_{2} \mathrm{CH}_{3}\right), 10.91\left(\mathrm{OCH}_{2} \mathrm{CH}_{2} \mathrm{CH}_{3}\right)$, $9.97\left(\mathrm{OCH}_{2} \mathrm{CH}_{2} \mathrm{CH}_{3}\right), 9.96\left(\mathrm{OCH}_{2} \mathrm{CH}_{2} \mathrm{CH}_{3}\right)$; HRMS-Positive: $\mathrm{m} / \mathrm{z}$ $\left[\mathrm{M}+\mathrm{NH}_{4}\right]^{+}$calcd. for $\mathrm{C}_{42} \mathrm{H}_{54} \mathrm{BrN}_{2} \mathrm{O}_{6}:$ : 761.3165; found 761.3157.

5-Menthyl carbamate-25,26,27,28-tetrapropoxycalix[4]arene (6) In an oven-dried 2-neck round-bottomed flask, compound 5 $(500 \mathrm{mg}, 0.823 \mathrm{mmol})$ was dissolved in DCM ( $40 \mathrm{~mL})$ and cooled 
to $0{ }^{\circ} \mathrm{C}$. Pyridine $(79.5 \mu \mathrm{L}, 0.987 \mathrm{mmol}, 1.2 \mathrm{eq})$ and menthyl chloroformate ( $209 \mu \mathrm{L}, 0.987 \mathrm{mmol}, 1.2 \mathrm{eq})$ were subsequently added and the mixture was warmed to room temperature. After $15 \mathrm{~min}$, the contents of the flask were poured into $\mathrm{H}_{2} \mathrm{O}(40 \mathrm{~mL})$ and extracted with DCM $(20 \mathrm{~mL} \times 3)$. The organic layers were then combined and first washed once with dilute $\mathrm{HCl}$ solution $(25 \mathrm{~mL}, 0.2 \mathrm{M})$ followed by brine $(25 \mathrm{~mL})$ and finally dried over $\mathrm{MgSO}_{4}$. The solvent was removed under reduced pressure and the crude product was purified via silica gel flash column chromatography (3:97 EtOAc:PET) to afford compound 6 as a colourless glass $(650 \mathrm{mg}, 98 \%) . \boldsymbol{R}_{f}=0.47(10: 90$ EtOAc:PET) $\mathbf{M p}=68-72{ }^{\circ} \mathrm{C},[\alpha]_{\mathrm{D}}^{20.6}=+23.0^{\circ}(\mathrm{c} 0.017, \mathrm{DCM}), \operatorname{IR}\left(\mathrm{ATR}, \mathbf{c m}^{-1}\right)$ : 2957, 2923 and $2872(\mathrm{C}-\mathrm{H}), 1697(\mathrm{C}=\mathrm{O}), 1454(\mathrm{C}=\mathrm{C}), 1210$ (C-O-C), 1006 and 966 (C-N), 757 (C-H); ${ }^{1} \mathrm{H}$ NMR (600 MHz, CHLOROFORM- $d$ ) $\delta$ ppm 6.84-6.39 (m, 11H, ArH), 6.06 (br. s, $1 \mathrm{H}, \mathrm{NHR}), 4.60\left(\mathrm{td},{ }^{3} J_{H H}=10.8,4.1 \mathrm{~Hz}, 1 \mathrm{H}, \mathrm{OCHhexyl}\right) 4.46\left(\mathrm{~d},{ }^{2} J_{H H}\right.$ $\left.=13.4 \mathrm{~Hz}, 2 \mathrm{H}, \mathrm{ArCH}_{2(a x .)} \mathrm{Ar}\right), 4.43\left(\mathrm{~d},{ }^{2} J_{\mathrm{HH}}=13.4 \mathrm{~Hz}, 2 \mathrm{H}\right.$, $\left.\mathrm{ArCH}_{2(a x .)} \mathrm{Ar}\right), 3.86\left(\mathrm{t},{ }^{3} \mathrm{~J}_{\mathrm{HH}} \stackrel{=}{=} 7.6 \mathrm{~Hz}, 4 \mathrm{H}, \mathrm{OCH}_{2} \mathrm{CH}_{2}\right), 3.82\left(\mathrm{t},{ }^{3} J_{\mathrm{HH}}=\right.$ $\left.7.3 \mathrm{~Hz}, 2 \mathrm{H}, \mathrm{OCH}_{2} \mathrm{CH}_{2}\right), 3.78\left(\mathrm{t}^{3}{ }^{3} \mathrm{HH}_{\mathrm{H}}=7.3 \mathrm{~Hz}, 2 \mathrm{H}, \mathrm{OCH}_{2} \mathrm{CH}_{2}\right), 3.16$ $\left(\mathrm{d},{ }^{2} J_{H H}=13.4 \mathrm{~Hz}, 2 \mathrm{H}, \mathrm{ArCH}_{2(e q) .)} \mathrm{Ar}\right), 3.12\left(\mathrm{~d},{ }^{2} J_{H H}=13.6 \mathrm{~Hz}, 2 \mathrm{H}\right.$, $\left.\mathrm{ArCH}_{2 \text { (eq.) }} \mathrm{Ar}\right), 2.10-2.04\left(\mathrm{~m}, 1 \mathrm{H}, \mathrm{CHC}_{3} \mathrm{H}_{7}\right), 1.97-1.85(\mathrm{~m}, 8 \mathrm{H}$, $\left.\mathrm{CH}_{3} \mathrm{CH}_{2} \mathrm{CH}_{3}\right), 1.72-1.66(\mathrm{~m}, 2 \mathrm{H}$, hexylH), 1.54-1.45 (m, $1 \mathrm{H}$, hexyl $H), 1.38-1.29(\mathrm{~m}, 1 \mathrm{H}$, hexyl $H), 1.12-0.83(\mathrm{~m}, 3 \mathrm{H}, \operatorname{hexyl} H)$, $1.03-0.95\left(\mathrm{~m}, 12 \mathrm{H}, \mathrm{OCH}_{2} \mathrm{CH}_{2} \mathrm{CH}_{3}\right), 0.91\left(2 \mathrm{xd},{ }^{3} J_{\mathrm{HH}}=6.7 \mathrm{~Hz}, 6 \mathrm{H}\right.$ $\left.\mathrm{CH}\left(\mathrm{CH}_{3}\right)_{2}\right), 0.79\left(\mathrm{~d}_{,}{ }^{2} J_{\mathrm{HH}}=7.0 \mathrm{~Hz}, 3 \mathrm{H}, \mathrm{CHCH}_{3}\right) .{ }^{13} \mathbf{C}\{\mathbf{1 H}\}(\mathbf{1 5 0 ~ M H z}$ CHLOROFORM- $d$ ) $\delta$ ppm 156.8 (ArC), 156.5 (NHCOO), 152.5 (ArC), $135.4(\operatorname{ArC}), 135.2(\operatorname{ArC}), 134.9(\operatorname{ArC}), 131.8(\operatorname{ArC}), 128.31$ $(\operatorname{ArC}), 128.27(\operatorname{ArC}), 127.9(\operatorname{ArC}), 122.92(\operatorname{ArC}), 122.91(\operatorname{ArC}), 121.5$ (ArC), 118.5 (ArC) $76.66\left(\mathrm{OCH}_{2} \mathrm{CH}_{2}\right), 76.64\left(\mathrm{OCH}_{2} \mathrm{CH}_{2}\right), 76.62$ $\left(\mathrm{OCH}_{2} \mathrm{CH}_{2}\right), 74.5$ (OCHhexyl), 47.4 (hexylC), 41.4 (hexylC), 34.3 (hexylC), $31.4\left(\mathrm{ArCH}_{2} \mathrm{Ar}\right), 31.1\left(\mathrm{ArCH}_{2} \mathrm{Ar}\right), 31.0$ $\left(\mathrm{ArCH} \mathrm{H}_{2} \mathrm{Ar}\right), 26.2\left(\mathrm{CH}\left(\mathrm{CH}_{3}\right)_{2}\right), 23.5$ (hexylC), $23.3\left(\mathrm{OCH}_{2} \mathrm{CH}_{2} \mathrm{CH}_{3}\right)$, $23.21\left(\mathrm{OCH}_{2} \mathrm{CH}_{2} \mathrm{CH}_{3}\right), 23.19\left(\mathrm{OCH}_{2} \mathrm{CH}_{2} \mathrm{CH}_{3}\right), 22.1\left(\mathrm{CH}\left(\mathrm{CH}_{3}\right)_{2}\right)$, $20.9\left(\mathrm{CH}\left(\mathrm{CH}_{3}\right)_{2}\right), 16.4($ hexylCH$), 10.43\left(\mathrm{OCH}_{2} \mathrm{CH}_{2} \mathrm{CH}_{3}\right), 10.41$ $\left(\mathrm{OCH}_{2} \mathrm{CH}_{2} \mathrm{CH}_{3}\right), 10.2\left(\mathrm{OCH}_{2} \mathrm{CH}_{2} \mathrm{CH}_{3}\right)$. HRMS-Positive: $\mathrm{m} / \mathrm{z}$ $\left[\mathrm{M}+\mathrm{NH}_{4}\right]^{+}$calcd. for $\mathrm{C}_{51} \mathrm{H}_{71} \mathrm{~N}_{2} \mathrm{O}_{6}$ : 807.5312; found 807.5313.

\section{4-Bromo-5-menthyl carbamate-25,26,27,28-tetrapropoxycalix [4]arene (7a and 7b)}

An oven-dried $5 \mathrm{~mL}$ Schlenk equipped with a magnetic stir bar was charged with NBS (14.9 mg, $0.084 \mathrm{mmol}, 1.1 \mathrm{eq}), \mathrm{PTSA} \cdot \mathrm{H}_{2} \mathrm{O}$ (7.2 mg, $0.038 \mathrm{mmol}, 0.5 \mathrm{eq}), \mathrm{Pd}(\mathrm{OAc})_{2}(0.9 \mathrm{mg}$, $0.004 \mathrm{mmol}$, $0.05 \mathrm{eq})$ and DCM $(1 \mathrm{~mL})$. The reaction vessel was subjected to five freeze pump thaw cycles before being flushed with argon and cooled to $-35{ }^{\circ} \mathrm{C}$. Once cooled, compound $10(60 \mathrm{mg}$, $0.078 \mathrm{mmol}$ ) was added at once and the reaction was left to stir at $-35^{\circ} \mathrm{C}$ for a further $4 \mathrm{~h}$. After the time had elapsed, the reaction contents were diluted with DCM $(10 \mathrm{~mL})$ and washed with a $10 \% \mathrm{HCl}$ solution $(15 \mathrm{~mL})$. The aqueous phase was extracted with DCM $(10 \mathrm{~mL} \times 3)$ before combining the organic phase and washing it once with sat. $\mathrm{NaHCO}_{3}(20 \mathrm{~mL})$ and finally brine $(20 \mathrm{~mL})$. The solution was finally dried over $\mathrm{MgSO}_{4}$ and concentrated under vacuo. The crude product was then purified via silica gel flash column chromatography (1:99 EtOAc:PET) to produce compounds $7 \mathbf{a}$ and $7 \mathbf{b}$ as an inseparable mixture of diastereomers (61 mg, $91 \%) . \boldsymbol{R}_{f}=0.27$ (4:96 EtOAc:PET); $\mathbf{M p}=$ 74-84 ${ }^{\circ} \mathrm{C}$; IR (ATR, cm $\left.^{-1}\right)$ : $3411(\mathrm{~N}-\mathrm{H}), 2957,2931$ and $2872(\mathrm{C}-\mathrm{H})$, $1732(\mathrm{C}=\mathrm{O}), 1509$ (arene), $1455(\mathrm{C}=\mathrm{C}), 1209$ and 1187 (C-O-C), 1005 and 966 (C-N), 756 (C-H); ${ }^{1} \mathrm{H}$ NMR (300 MHz, CHLOROFORM- $d$ ) $\delta$ ppm 7.95-7.84 (m, 1H, ArH - meta to Br), 7.20-7.02 $(\mathrm{m}, 2 \mathrm{H}, \mathrm{ArH}), 6.91+6.89\left(2 \times \mathrm{t},{ }^{3} \mathrm{~J}_{\mathrm{HH}}=7.3 \mathrm{~Hz}, 1 \mathrm{H}, \mathrm{ArH}\right), 6.36-6.06$ $(\mathrm{m}, 6 \mathrm{H}, \mathrm{ArH}), 4.77-4.61(\mathrm{~m}, 1 \mathrm{H}$, menthyl-OCH), 4.48-4.36 (m, 4H, $\left.\mathrm{ArCH}_{2(a x)} \mathrm{Ar}\right), 4.10-3.79\left(\mathrm{~m}, 4 \mathrm{H}, \mathrm{OCH}_{2} \mathrm{CH}_{2}+1 \mathrm{H}, \mathrm{ArCH}_{2(e q) .)} \mathrm{Ar}\right)$, 3.72-3.61 (m, 4H, OCH $\left.\mathrm{CH}_{2}\right), 3.24-3.09\left(\mathrm{~m}, 3 \mathrm{H}, \mathrm{ArCH}_{2(\text { eq. })} \mathrm{Ar}\right)$,
2.23-2.03 (m, 1H, menthyl $\mathrm{H}), 2.01-1.80\left(\mathrm{~m}, 8 \mathrm{H}, \mathrm{OCH}_{3} \mathrm{CH}_{2} \mathrm{CH}_{3}\right)$, $1.72\left(\right.$ app d, ${ }^{2} J_{H H}=11.6 \mathrm{~Hz}, 2 \mathrm{H}$, menthyl- $\left.H\right), 1.51-1.38(\mathrm{~m}, 1 \mathrm{H}$, menthyl- $H), 1.51-0.74\left(\mathrm{~m}, 27 \mathrm{H}\right.$, menthyl- $H^{\prime} \mathrm{s}$, menthyl- $\mathrm{CH}_{3}$ 's, $\left.\mathrm{OCH}_{3} \mathrm{CH}_{2} \mathrm{CH}_{3}\right) ;{ }^{13} \mathrm{C}\{\mathbf{1 H}\}(75 \mathbf{M H z}, \mathrm{CHLOROFORM}-d)^{*} \delta$ ppm $158.1+157.1+155.3$ (ArC-OPr), 154.4 (ArC-OPr Br ring), 153.6 $(\mathrm{C}=\mathrm{O}), 139.2+139.0+137.5+137.2+136.7+136.4+133.5+$ $132.7+132.5+132.4+132.2(\mathrm{ArC}), 131.3(\mathrm{ArCH}), 130.7+130.6$ (ArC-Br), $129.0+127.9+127.7+127.5+127.3+126.7+126.5+$ $122.3+121.9(\mathrm{ArCH}), 120.8(\mathrm{ArCH}-$ meta to $\mathrm{Br}), 114.4+113.9$ (ArC), $77.0+76.6\left(\mathrm{OCH}_{2} \mathrm{CH}_{2} \mathrm{CH}_{3}\right), 75.5+75.4$ (menthyl-OCH), 47.3 (menthyl- $\mathrm{CH}$ ), 41.4 (menthyl- $\mathrm{CH}_{2}$ ), 34.4 (menthyl- $\mathrm{CH}_{2}$ ), $31.6+31.0+30.3\left(\mathrm{ArCH}_{2} \mathrm{Ar}\right), 26.4$ (menthyl- $\left.\mathrm{CH}\right), 23.6+23.0$ (menthyl- $\mathrm{CH}_{2}+\mathrm{OCH}_{2} \mathrm{CH}_{2} \mathrm{CH}_{3}$ ), $22.2+21.0+16.7$ (menthyl$\left.\mathrm{CH}_{3}{ }^{\prime} \mathrm{s}\right), 10.9+9.9\left(-\mathrm{OCH}_{2} \mathrm{CH}_{2} \mathrm{CH}_{3}\right)$; HRMS-Positive: $\mathrm{m} / \mathrm{z}$ $\left[\mathrm{M}+\mathrm{NH}_{4}\right]^{+}$calcd. for $\mathrm{C}_{51} \mathrm{H}_{70} \mathrm{BrN}_{2} \mathrm{O}_{6}:$ 885.4417; found 885.4427.

\section{4-Bromo-5-amino-25,26,27,28-tetrapropoxycalix[4]arene (8)}

In an oven-dried 2-neck round-bottomed flask equipped with a reflux condenser and magnetic stir bar was charged with calixarene $7 \mathbf{a}$ and $7 \mathbf{b}(110 \mathrm{mg}, 0.127 \mathrm{mmol})$, a $1 \mathrm{M}$ solution of TBAF in THF, $(1.27 \mathrm{~mL}, 10 \mathrm{eq})$ and THF $(10 \mathrm{~mL})$. The contents were heated to reflux and left to stir for $36 \mathrm{~h}$. After the time had elapsed, the solution was concentrated and then diluted with EtOAc $(15 \mathrm{~mL})$ before being washed with $\mathrm{H}_{2} \mathrm{O}(10 \mathrm{~mL} \times 6)$ and finally brine $(10 \mathrm{~mL})$. The liquid was dried over $\mathrm{MgSO}_{4}$ and concentrated via reduced pressure. The crude product was then purified firstly via silica gel flash column chromatography (EtOAc:PET 1:9) and then heated at $80{ }^{\circ} \mathrm{C}$ under reduced pressure for $8 \mathrm{~h}$ to afford compound 8 as a yellow solid $(72 \mathrm{mg}, 83 \%)$. $\boldsymbol{R}_{f}=0.33$ (5:95 EtOAc:PET); $\mathbf{M p}=130-136{ }^{\circ} \mathrm{C} ;$ IR $\left(\right.$ ATR, $\left.\mathbf{~ c m}^{-1}\right)$ : 1264 (C-N), 731 (C-H), 703 (C-H); ${ }^{1}$ H NMR (600 MHz, CHLOROFORM- $d$ ) $\delta$ ppm $7.09\left(\mathrm{~d},{ }^{3} J_{H H}=7.4 \mathrm{~Hz}, 2 \mathrm{H}, \mathrm{ArH}\right), 6.91\left(\mathrm{t},{ }^{3} J_{H H}=7.4\right.$ $\mathrm{Hz}, 1 \mathrm{H}, \operatorname{ArH}), 6.60$ (s, 1H, ArH), 6.30-6.12 (m, 6H, ArH), 4.45 (d, $\left.{ }^{2} J_{H H}=13.3 \mathrm{~Hz}, 1 \mathrm{H}, \mathrm{ArCH}(2 a x.) \mathrm{Ar}\right), 4.44\left(\mathrm{~d},{ }^{2} J_{\mathrm{HH}}=13.3 \mathrm{~Hz}, 1 \mathrm{H}\right.$, $\left.\mathrm{ArCH}_{2(a x .)} \mathrm{Ar}\right), 4.37\left(\mathrm{~d},{ }^{2} J_{\mathrm{HH}}=13.6 \mathrm{~Hz}, 2 \mathrm{H}, \mathrm{ArCH}_{2(a \mathrm{x} .)} \mathrm{Ar}\right), 4.06-3.79$ $\left(\mathrm{m}, 4 \mathrm{H}, \mathrm{OCH}_{2} \mathrm{CH}_{2}, 2 \mathrm{H}, \mathrm{NH}_{2}\right), 3.83\left(\mathrm{~d},{ }^{2} J_{\mathrm{HH}}=13.6 \mathrm{~Hz}, 1 \mathrm{H}\right.$, $\left.\mathrm{ArCH}_{2(\text { eq. })} \mathrm{Ar}\right), 3.75-3.62\left(\mathrm{~m}, 4 \mathrm{H}, \mathrm{OCH}_{2} \mathrm{CH}_{2}\right), 3.16\left(\mathrm{~d},{ }^{2} J_{\mathrm{HH}}=13.4 \mathrm{~Hz}\right.$, $\left.1 \mathrm{H}, \mathrm{ArCH}_{2(\mathrm{eq}) .)} \mathrm{Ar}\right), 3.15\left(\mathrm{~d},{ }^{2} J_{\mathrm{HH}}=13.4 \mathrm{~Hz}, 1 \mathrm{H}, \mathrm{ArCH}_{2(\text { eq. })} \mathrm{Ar}\right), 3.03(\mathrm{~d}$, $\left.{ }^{2} J_{H H}=13.3 \mathrm{~Hz}, 1 \mathrm{H}, \mathrm{ArCH}{ }_{2(e q)} \mathrm{Ar}\right), 2.04-1.82\left(\mathrm{~m}, 8 \mathrm{H}, \mathrm{CH}_{3} \mathrm{CH}_{2} \mathrm{CH}_{3}\right)$, $1.10\left(\mathrm{t},{ }^{3} J_{\mathrm{HH}}=7.4 \mathrm{~Hz}, 6 \mathrm{H}, \mathrm{CH}_{2} \mathrm{CH}_{2} \mathrm{CH}_{3}\right), 0.90\left(\mathrm{t},{ }^{3} J_{\mathrm{HH}}=7.4 \mathrm{~Hz}, 3 \mathrm{H}\right.$, $\left.\mathrm{CH}_{2} \mathrm{CH}_{2} \mathrm{CH}_{3}\right), 0.89\left(\mathrm{t},{ }^{3} \mathrm{~J}_{\mathrm{HH}}=7.4 \mathrm{~Hz}, 3 \mathrm{H}, \mathrm{CH}_{2} \mathrm{CH}_{2} \mathrm{CH}_{3}\right) ;{ }^{13} \mathrm{C}\{\mathbf{1 H}\}$ (150 MHz, CHLOROFORM- $d$ ) $\delta$ ppm 158.1 (ArC), 155.3 (ArC), 150.9 (ArC), $139.0(\operatorname{ArC}), 137.6(\operatorname{ArC}), 137.3(\operatorname{ArC}), 137.1(\operatorname{ArC})$, $136.6(\mathrm{ArC}), 133.5(\mathrm{ArC}), 133.3(\mathrm{ArC}), 132.6(\mathrm{ArC}), 129.0(\mathrm{ArC})$, 128.9 (ArC), $127.7(\operatorname{ArC}), 127.6(\operatorname{ArC}), 127.3(\operatorname{ArC}), 126.8(\operatorname{ArC})$, 122.2 (ArC), 121.8 (ArC), $115.7(\mathrm{ArC}), 110.4(\mathrm{ArC}), 77.06$ $\left(\mathrm{OCH}_{2} \mathrm{CH}_{2}\right), 76.96\left(\mathrm{OCH}_{2} \mathrm{CH}_{2}\right), 76.95\left(\mathrm{OCH}_{2} \mathrm{CH}_{2}\right), 76.55$ $\left(\mathrm{OCH}_{2} \mathrm{CH}_{2}\right), 31.18\left(\mathrm{ArCH} \mathrm{Ar}_{2} \mathrm{Ar}\right), 31.11\left(\mathrm{ArCH}_{2} \mathrm{Ar}\right), 30.89\left(\mathrm{ArCH} \mathrm{H}_{2} \mathrm{Ar}\right)$, $29.93\left(\mathrm{ArCH} \mathrm{H}_{2} \mathrm{Ar}\right), 23.66\left(\mathrm{OCH}_{2} \mathrm{CH}_{2} \mathrm{CH}_{3}\right), 23.62\left(\mathrm{OCH}_{2} \mathrm{CH}_{2} \mathrm{CH}_{3}\right)$, $23.10\left(\mathrm{OCH}_{2} \mathrm{CH}_{2} \mathrm{CH}_{3}\right), 22.97\left(\mathrm{OCH}_{2} \mathrm{CH}_{2} \mathrm{CH}_{3}\right), 10.95$ $\left(\mathrm{OCH}_{2} \mathrm{CH}_{2} \mathrm{CH}_{3}\right), 10.94\left(\mathrm{OCH}_{2} \mathrm{CH}_{2} \mathrm{CH}_{3}\right), 10.01\left(\mathrm{OCH}_{2} \mathrm{CH}_{2} \mathrm{CH}_{3}\right)$, $9.99\left(\mathrm{OCH}_{2} \mathrm{CH}_{2} \mathrm{CH}_{3}\right)$. HRMS-Positive: $\mathrm{m} / \mathrm{z}[\mathrm{M}+\mathrm{H}]^{+}$calcd. for $\mathrm{C}_{40} \mathrm{H}_{49} \mathrm{BrNO}_{4}$ : 686.2845; found 686.2849.

\section{Acknowledgements}

This work was supported by the National Research Foundation (Grant CPRR160428163281) and Stellenbosch University. K.J.V. is grateful to the DST/NRF for an MSc innovation scholarship and L.H. to the NRF for postdoctoral funding. We would also like to thank Dr. J. Brand and Ms. E Malherbe for the NMR spectroscopic service and Dr. M Stander and Mr. M Taylor for the mass spectroscopic service.

* Due to diastereomers, signals were difficult to assign accurately. Assignments based on 2D NMR spectroscopy only where it was clear. 


\section{${ }^{5}$ ORCID iDs}

K.J. Visagie:

L. Hodson:

G.E. Arnott:

\section{References}

1 A. Szumna, Inherently chiral concave molecules - from synthesis to applications, Chem. Soc. Rev., 2010, 39(11), 4274-4285.

2 G.E. Arnott, Inherently chiral calixarenes: synthesis and applications, Chem. - A Eur. J., 2018, 24(8), 1744-1754

3 L.X. Dai, T. Tu, S.L. You, W.P. Deng and X.L. Hou, Asymmetric catalysis with chiral ferrocene ligands, Acc. Chem. Res., 2003, 36(9), 659-667.

4 S.A. Herbert and G.E. Arnott, An asymmetric ortholithiation approach to inherently chiral calix[4]arenes, Org. Lett., 2009, 11(21), 4986-4989.

5 S.A. Herbert and G.E. Arnott, Synthesis of inherently chiral calix[4]arenes: stereocontrol through ligand choice, Org. Lett., 2010, 12(20), 4600-4603.

6 S.A. Herbert, D.C. Castell, D.C. Clayden and G.E. Arnott, Manipulating the diastereoselectivity of ortholithiation in planar chiral ferrocenes, Org. Lett., 2013, 15(13), 3334-3337.

7 D.C. Castell, N. Lesotho, V.I. Nikolayenko and G.E. Arnott, Inherently chiral calix[4]arenes: a chiral sulfoxide as an ortholithiation director, European J. Org. Chem., 2017, 2017(29), 4328-4333.

8 F.M. Moghaddam, G. Tavakoli, B. Saeednia, P. Langer and B. Jafari, Palladium-catalyzed carbamate-directed regioselective halogenation: a route to halogenated anilines, J. Org. Chem., 2016, 81(9), 3868-3876.

9 G.E. Arnott, Inherently chiral calixarenes: synthesis and applications, Chem. - A Eur. J., 2018, 24(8), 1744-1754.

10 M.C. Davis, Chlorination of aniline and methyl carbanilate by N-chlorosuccinimide and synthesis of 1,3,5-trichlorobenzene, Synth. Commun., 2009, 39(6), 1100-1108.

11 P.V.N. Reddy, B. Banerjee and M. Cushman, Efficient total synthesis of ammosamide B, Org. Lett., 2010, 12(13), 3112-3114.

12 A. Roth, H. Li, C. Anorma and J. Chan, A reaction-based fluorescent probe for imaging of formaldehyde in living cells, J. Am. Chem. Soc., 2015, 137(34), 10890-10893.

13 D.L. Boger, R.J. Wysocki, S.A. Munk, T. Ishizaki, P.A. Kitos and O. Suntornwat, Total synthesis and evaluation of $( \pm)$-N-(tert-butyloxy-
carbonyl)-CBI, ( \pm )-CBI-CDPI1, and ( \pm )-CBI-CDPI2: CC-1065 functional agents incorporating the equivalent $1,2,9,9$ a-tetrahydrocycloprop[1,2-c]benz[1,2-e]indol-4-one (CBI) left-hand subunit, J. Am. Chem. Soc., 1989, 111(16), 6461-6463.

$14 \mathrm{M}$. Guyonnet and O. Baudoin, Synthesis of tricyclic nitrogen heterocycles by a sequence of palladium-catalyzed N-H and C(sp 3)-H arylations, Org. Lett., 2012, 14(1), 398-401.

15 R.A. Fujimoto, L.W. Mcquire, L.G. Monovich, B.B. Mugrage, D.T. Parker, J.H. Van Duzer and S. Wattanasin, Substituted amino phenylacetic acids, derivatives thereof, their preparation and their use as cyclooxygenase 2 (cox-2) inhibitors, WO 2004/048314 A1, 2004.

16 N. Uhlig and C.J. Li, Aniline carbamates: a versatile and removable motif for palladium-catalyzed directed c-h activation, Chem. - A Eur. J., 2014, 20(38), 12066-12070.

17 X. Shi and D. Shi, Recent advances in transition-metal-catalyzed halides formation, Curr. Org. Chem., 2018, 22(23), 2229-2255.

18 C. Que, N. Chen and J. Xu, Application of carbamates in the C-H bond activation, Prog. Chem., 2018, 30(2-3), 139-155.

19 R. Das and M. Kapur, Transition-metal-catalyzed site-selective C-H halogenation reactions, Asian J. Org. Chem., 2018, 7(8), 1524-1541.

20 C. Sambiagio, D. Schönbauer, R. Blieck, T. Dao-Huy, G. Pototschnig, P. Schaaf, T. Wiesinger, M.F. Zia, J. Wencel-Delord, T. Besset, B.U.W. Maes and M. Schnürch, A comprehensive overview of directing groups applied in metal-catalysed C-H functionalisation chemistry, Chem. Soc. Rev., 2018, 47(17), 6603-6743.

21 A.M.A. Van Wageningen, E. Snip, W. Verboom, D.N. Reinhoudt and $\mathrm{H}$. Boerrigter, Synthesis and application of iso(thio)cyanatefunctionalized calix[4]arenes, Liebigs Ann., 1997, 11, 2235-2245.

22 C.D. Jurisch and G.E. Arnott, Attempted synthesis of a meta -metalated calix[4]arene, Beilstein J. Org. Chem., 2019, 15(1), 1996-2002.

23 S. Pinchas and D. Ben-Ishai, The carbonyl absorption of carbamates and 2-oxazolidones in the infrared region, J. Am. Chem. Soc., 1957, 79(15), 4099-4104.

24 U. Jacquemard, V. Bénéteau, M. Lefoix, S. Routier, J.Y. Mérour and G. Coudert, Mild and selective deprotection of carbamates with Bu $4 \mathrm{NF}$, Tetrahedron, 2004, 60(44), 10039-10047.

25 P.M. Esch, H. Hiemstra and W.N. Speckamp, Oxazinone versus allene formation in the reaction of $\mathrm{N}$-alkoxycarbonyliminium ions with propargyltrimethylsilane, Tetrahedron Lett., 1988, 29(3), 367-370. 


\section{Supplementary material to:}

K.J. Visagie, L. Hodson and G.E. Arnott,

C-H activation: a Critical Evaluation of a Published Method and its Application Towards Inherently Chiral Calix[4]arenes,

S. Afr. J. Chem., 2020, 73, 15-21. 


\section{Supplementary Information for \\ Investigation into a $\mathrm{C}-\mathrm{H}$ activation pathway towards inherently chiral calix[4]arenes}

Kevin J. Visagie, Luke Hodson and Gareth E. Arnott*

Department of Chemistry and Polymer Science, Stellenbosch University, Private Bag X1, Matieland, 7602, South Africa

\section{Contents:}

NMR, IR and HRMS Spectra for all new compounds synthesized and also References for halogenation reactions prior to 2016 


\section{5-Methyl carbamate-25,26,27,28-tetrapropoxycalix[4]arene (4)}

IIVisagie\Chapter 3 - Mono Carbamate Calixarenes\(8) 5-Methyl carbamate-25,26,27,28-tetrapropoxy-calix[4]arene

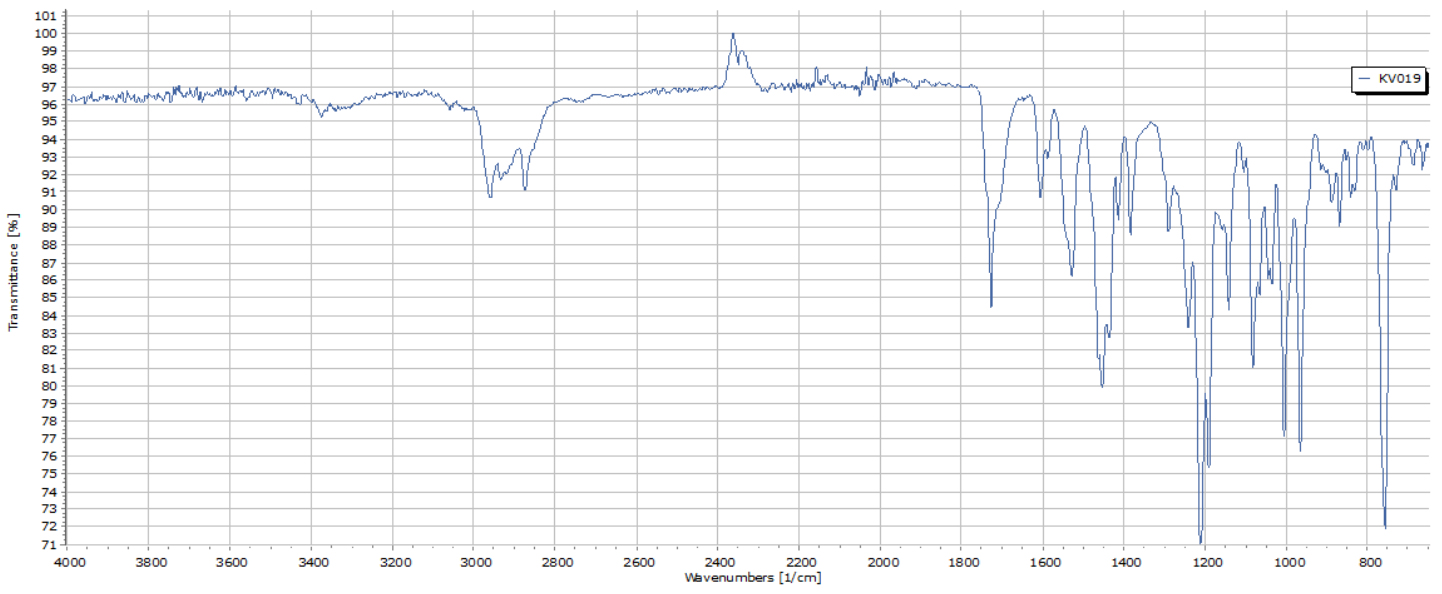

Figure 1. IR spectrum (ATR) for 5-methyl carbamate-25,26,27,28-tetrapropoxycalix[4]arene 4.

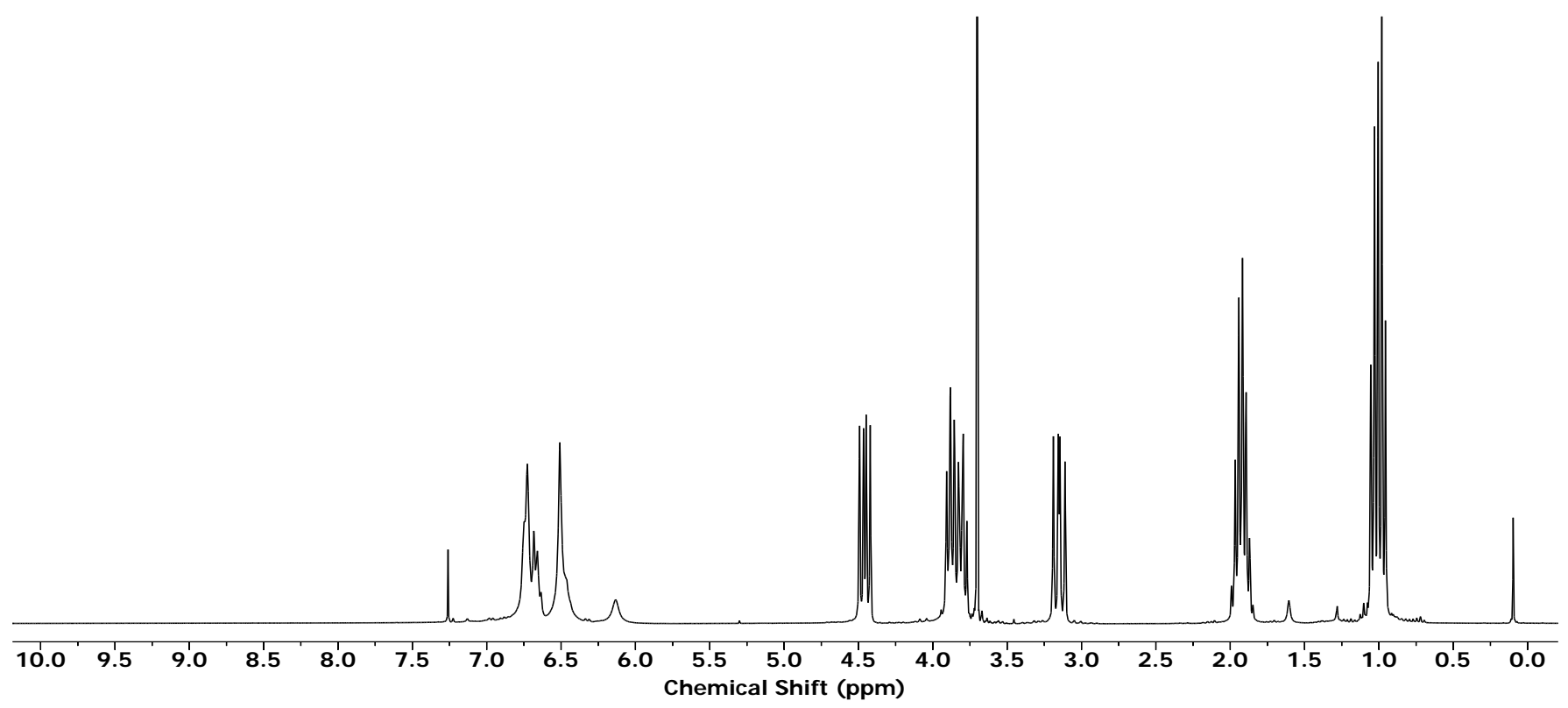

Figure 2. ${ }^{1} \mathrm{H} N \mathrm{NM}$ spectrum $\left(300 \mathrm{MHz}, \mathrm{CDCl}_{3}\right.$ ) for 5-methyl carbamate-25,26,27,28-tetrapropoxycalix[4]arene 4.

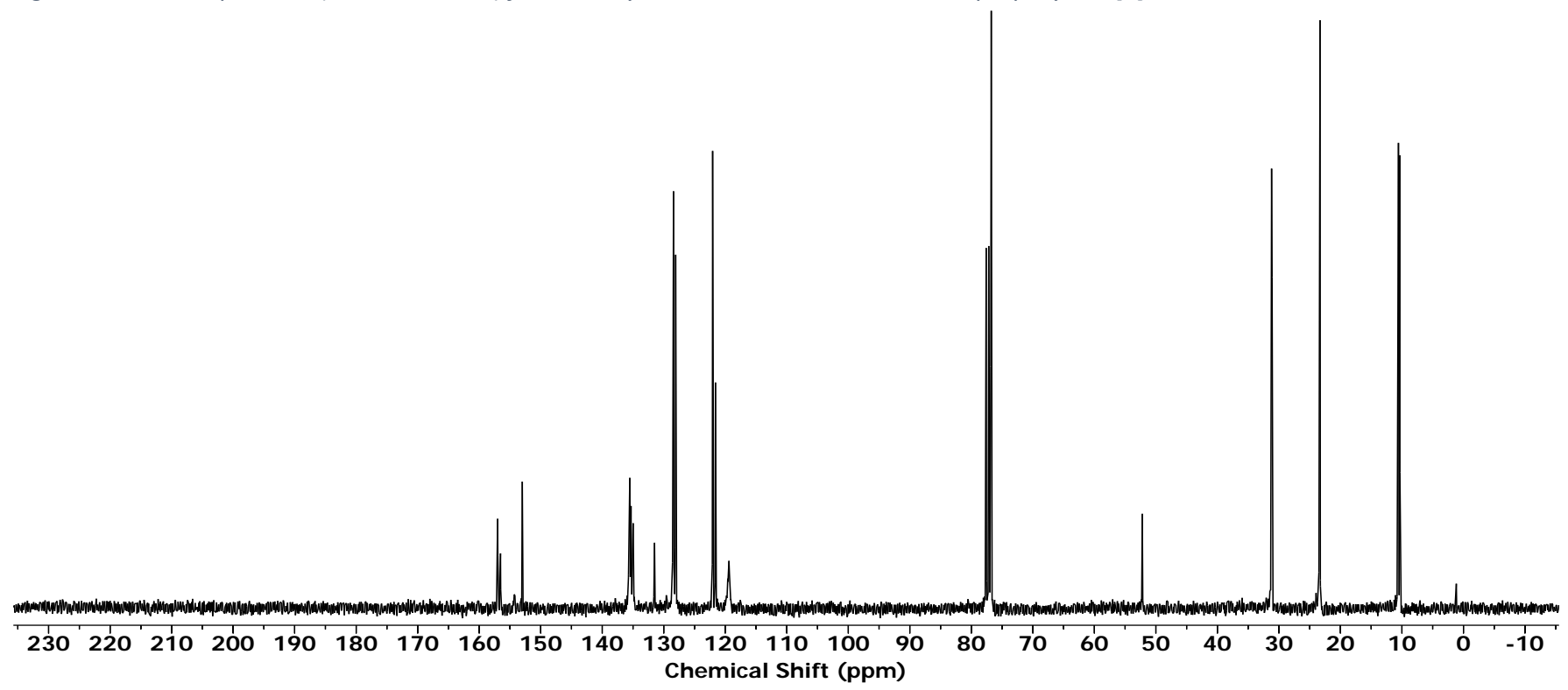

Figure 3. ${ }^{13} \mathrm{CNMR}$ spectrum (75MHz, $\left.\mathrm{CDCl}_{3}\right)$ for 5-methyl carbamate-25,26,27,28-tetrapropoxycalix[4]arene 4. 


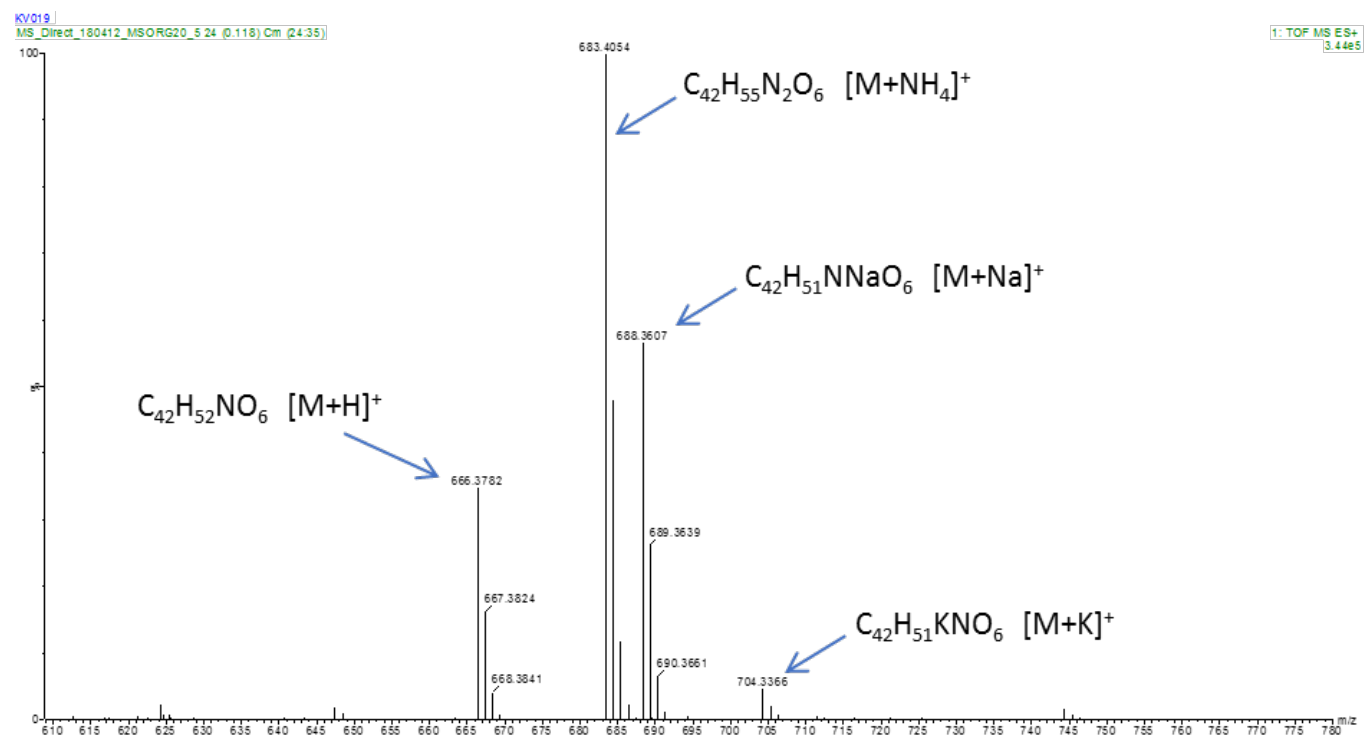

Figure 4. HRMS spectrum (ESI+) for 5-methyl carbamate-25,26,27,28-tetrapropoxycalix[4]arene 4.

\section{4-Bromo-5-methyl carbamate-25,26,27,28-tetrapropoxycalix[4]arene (5)}

IIVisagie\Chapter 3 - Mono Carbamate Calixarenes\(9) 4-Bromo-5-Methyl carbamate-25,26,27,28-tetrapropoxy-calix[4]arene

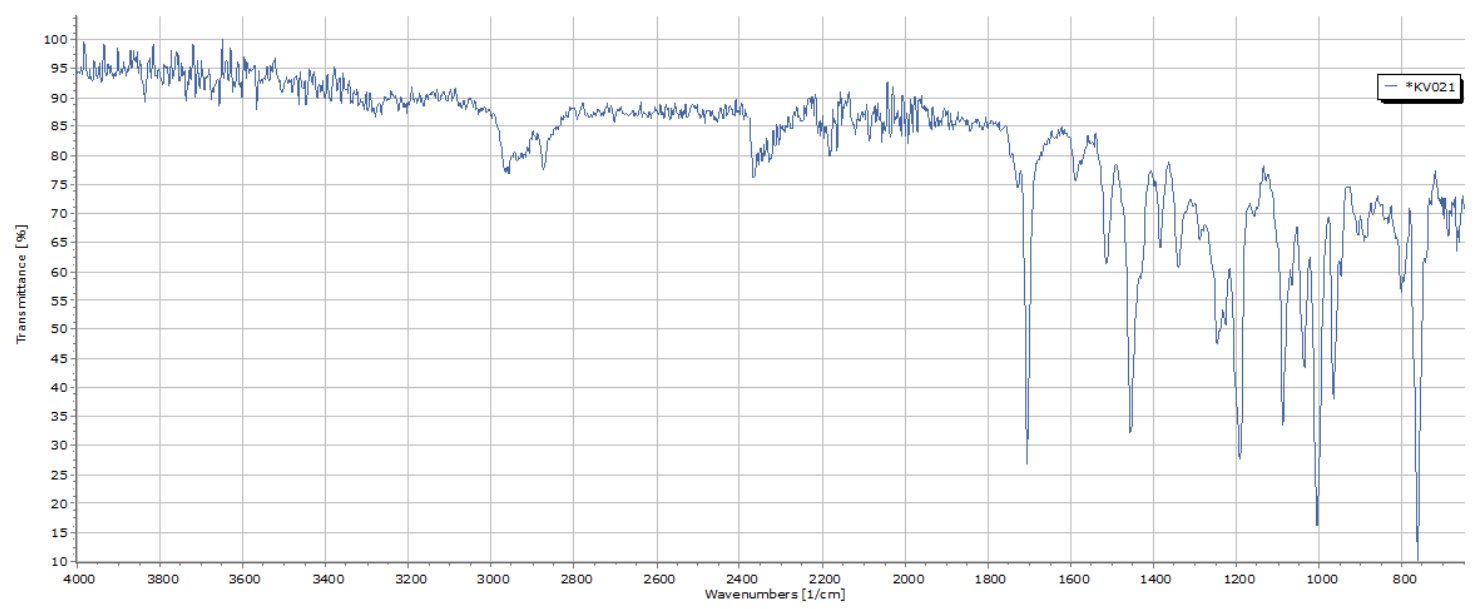

Figure 5. IR spectrum (ATR) for (士)-6-bromo-5-methyl carbamate-25,26,27,28-tetrapropoxycalix[4]arene 5

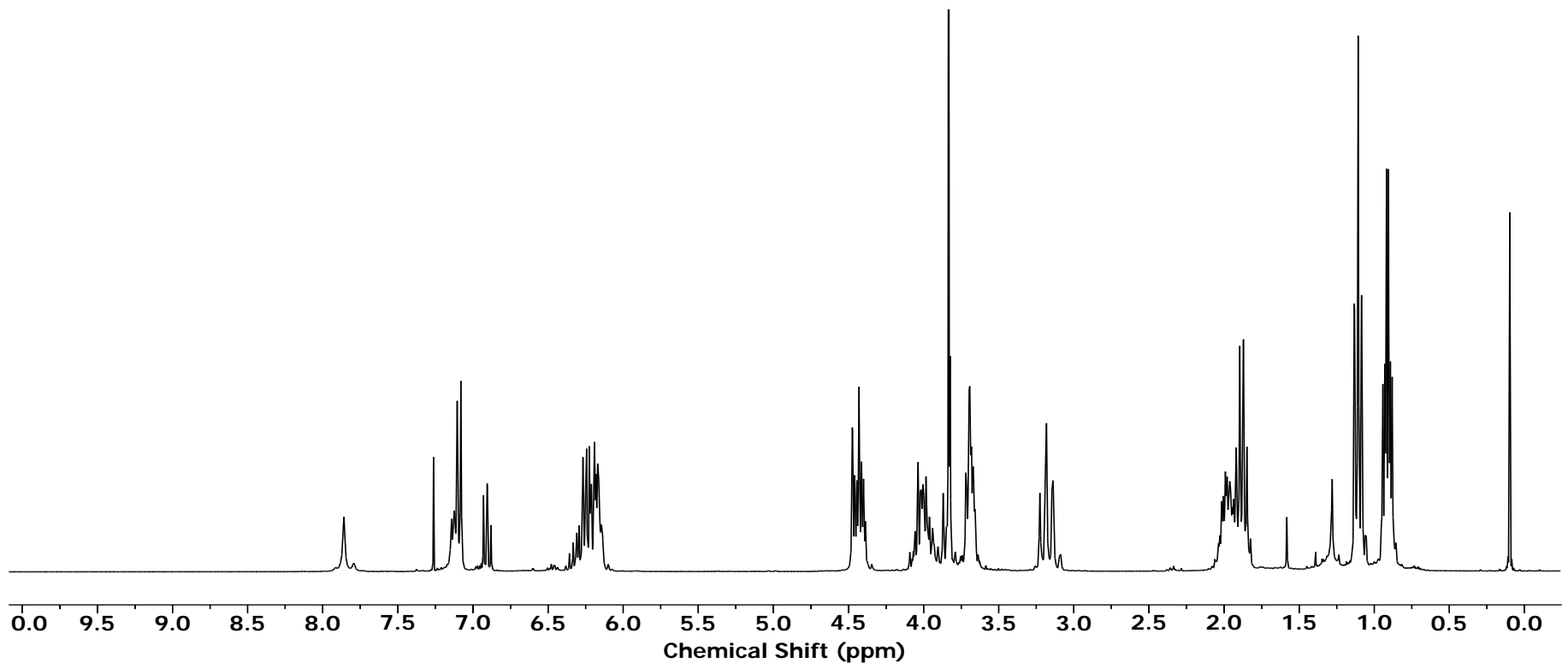




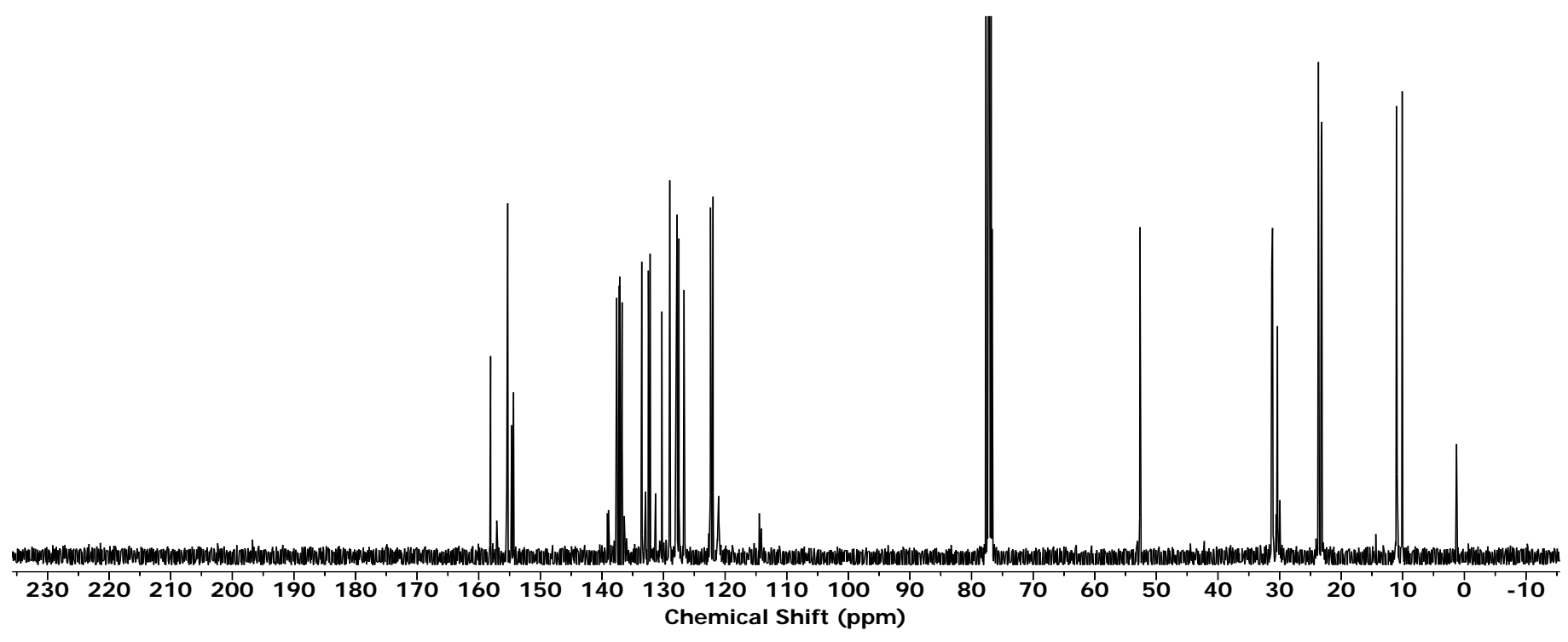

Figure 7. ${ }^{13} \mathrm{C} \mathrm{NMR}$ spectrum $\left(75 \mathrm{MHz}, \mathrm{CDCl}_{3}\right)$ for ( \pm )-6-bromo-5-methyl carbamate-25,26,27,28-tetrapropoxycalix[4]arene 5

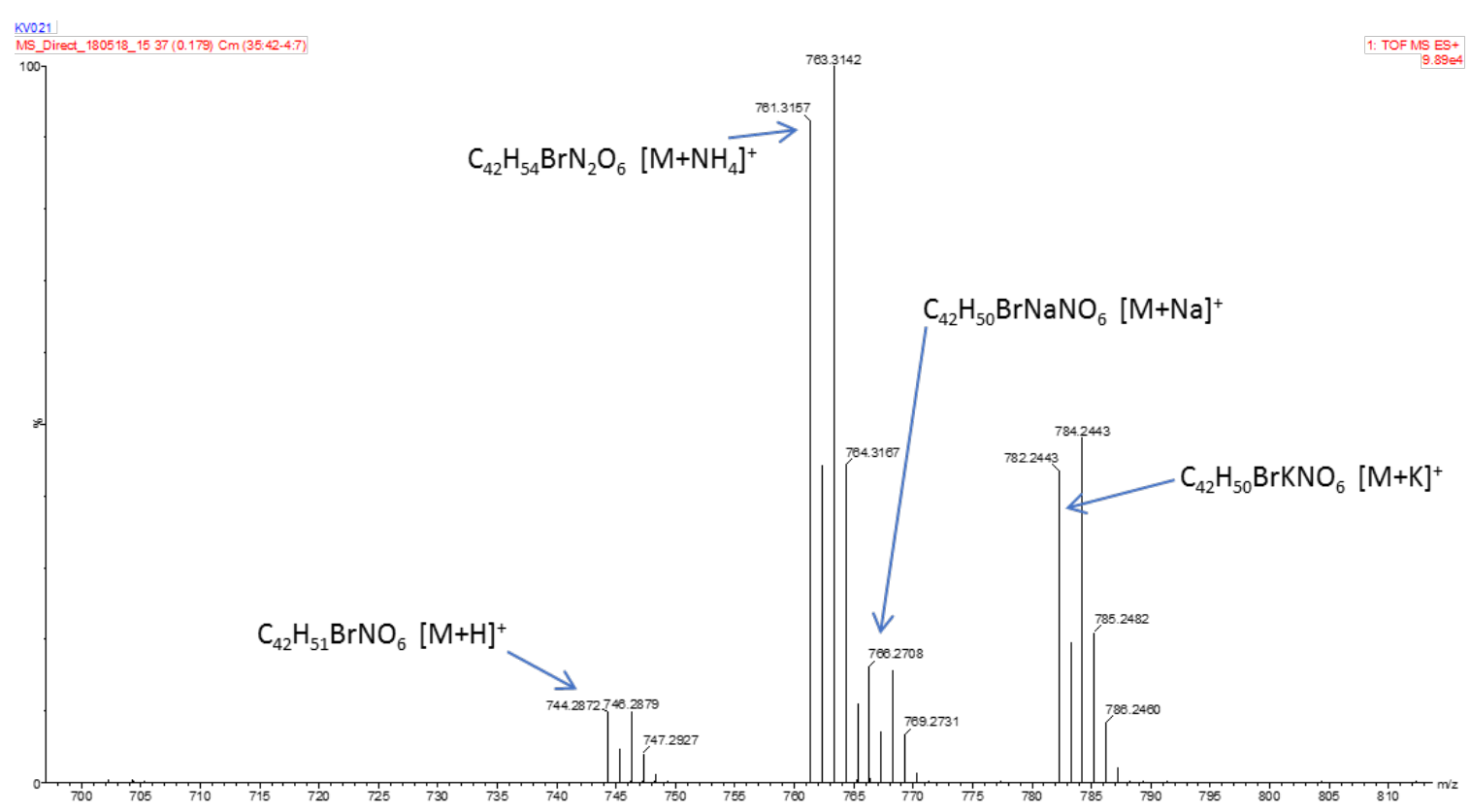

Figure 8. HRMS spectrum (ESI+) for ( \pm )-6-bromo-5-methyl carbamate-25,26,27,28-tetrapropoxycalix[4]arene 5

\section{5-Menthyl carbamate-25,26,27,28-tetrapropoxycalix[4]arene (6)}

IIVisagie \Chapter 3 - Mono Carbamate Calixarenes $\backslash(10)$ 5-Menthyl carbamate-25,26,27,28-tetrapropoxy-calix[4]arene

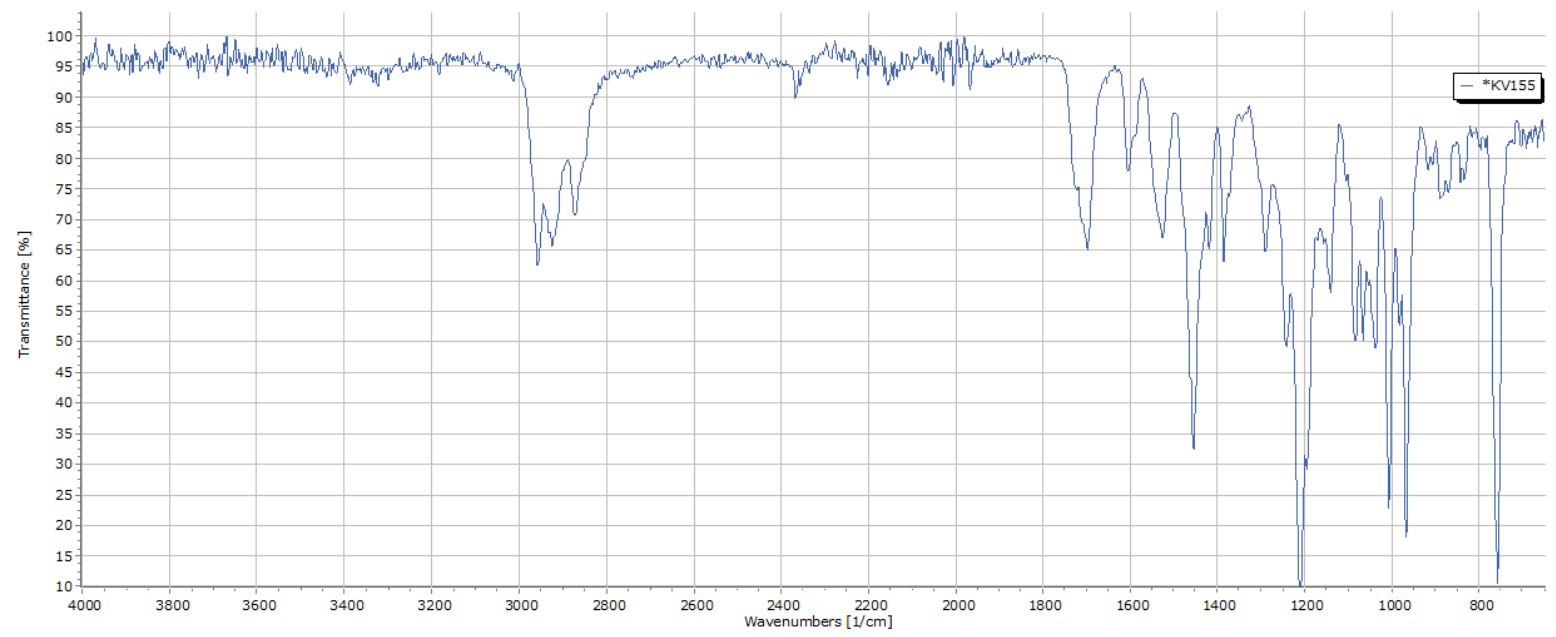

Figure 9. IR spectrum (ATR) for 5-menthyl carbamate-25,26,27,28-tetrapropoxycalix[4]arene 6 


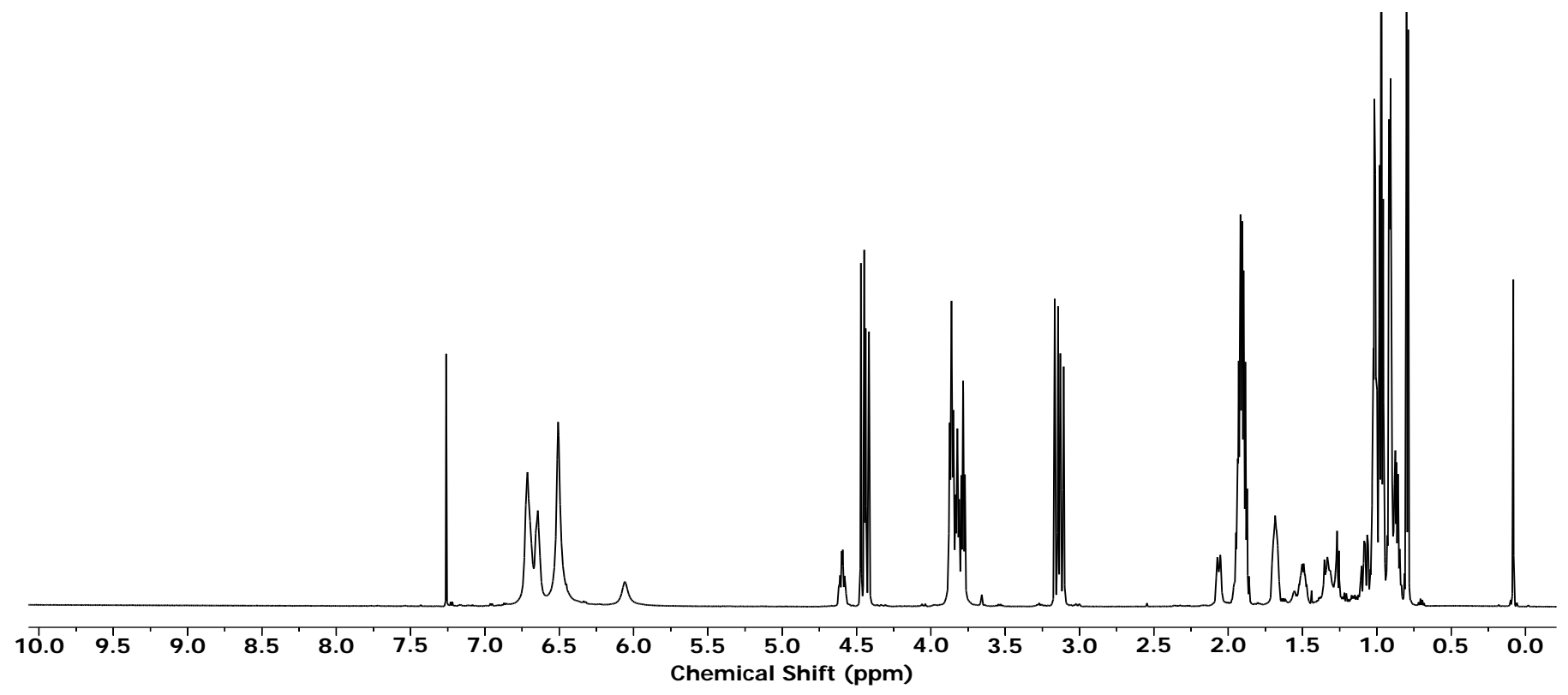

Figure 10. ${ }^{1} \mathrm{H} N \mathrm{NR}$ spectrum $\left(600 \mathrm{MHz}, \mathrm{CDCl}_{3}\right)$ for 5-menthyl carbamate-25,26,27,28-tetrapropoxycalix[4]arene 6

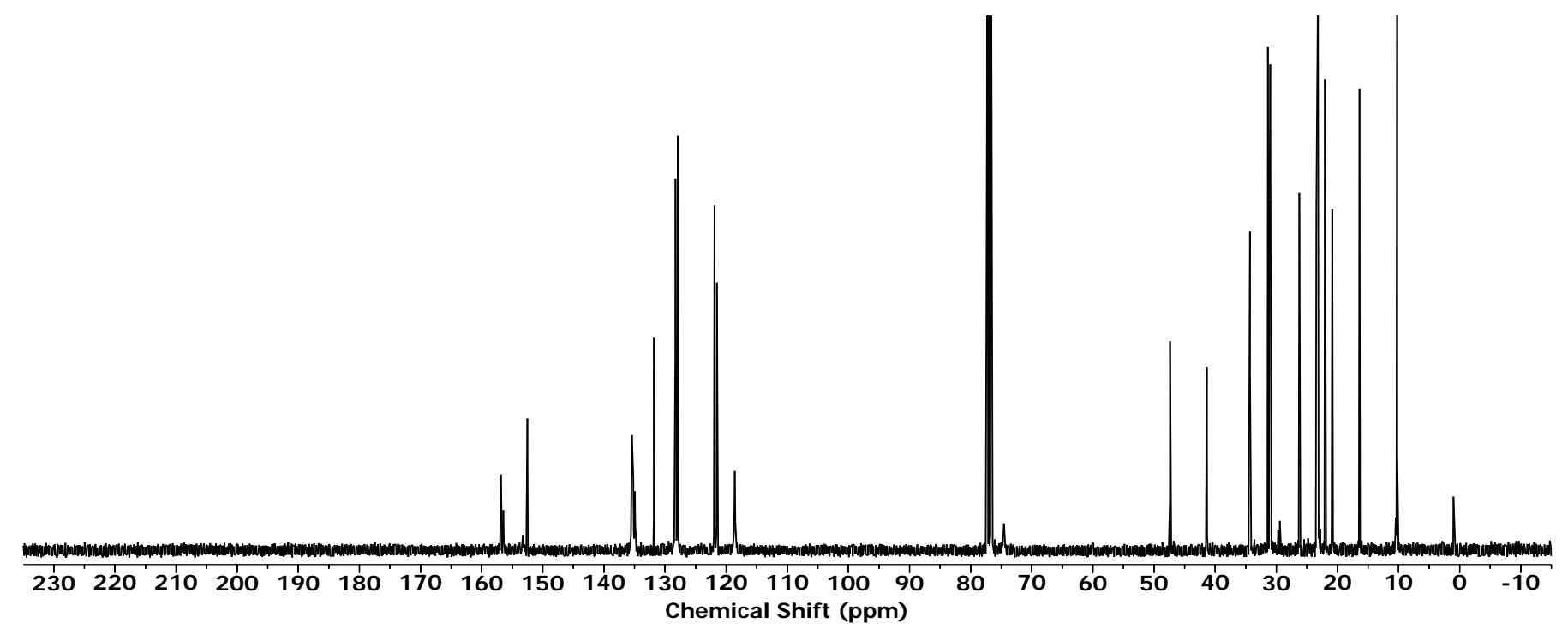

Figure 11. ${ }^{13} \mathrm{C} \mathrm{NMR}$ spectrum $\left(150 \mathrm{MHz}, \mathrm{CDCl}_{3}\right)$ for 5-menthyl carbamate-25,26,27,28-tetrapropoxycalix[4]arene 6

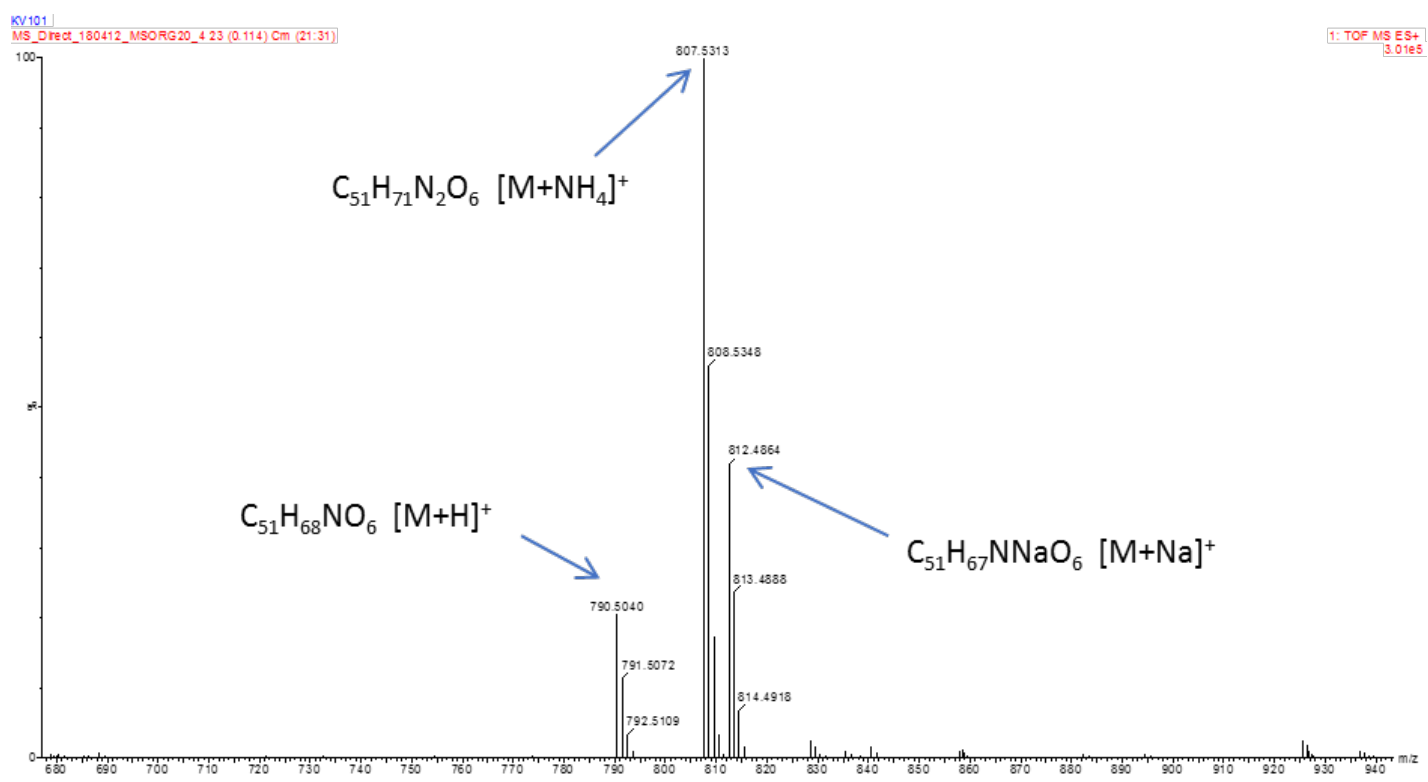

Figure 12. HRMS spectrum (ESI+) for 5-menthyl carbamate-25,26,27,28-tetrapropoxycalix[4]arene 6 
IIVisagie $\backslash$ Chapter 3 - Mono Carbamate Calixarenes $\backslash(11)$ 4-Bromo-5-menthyl carbamate-25,26,27,28-tetrapropoxy calix[4]arene

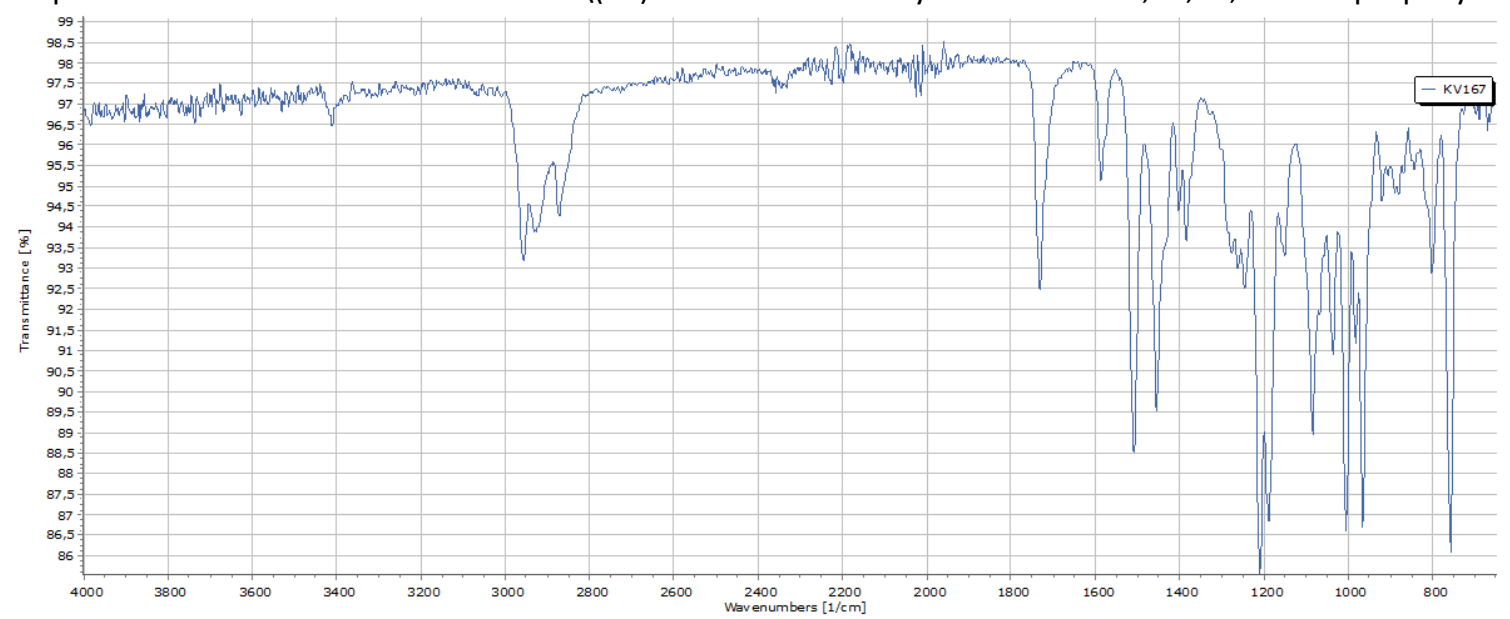

Figure 13. IR spectrum (ATR) for 4-bromo-5-menthyl carbamate-25,26,27,28-tetrapropoxycalix[4]arene 7a and $7 \mathbf{b}$

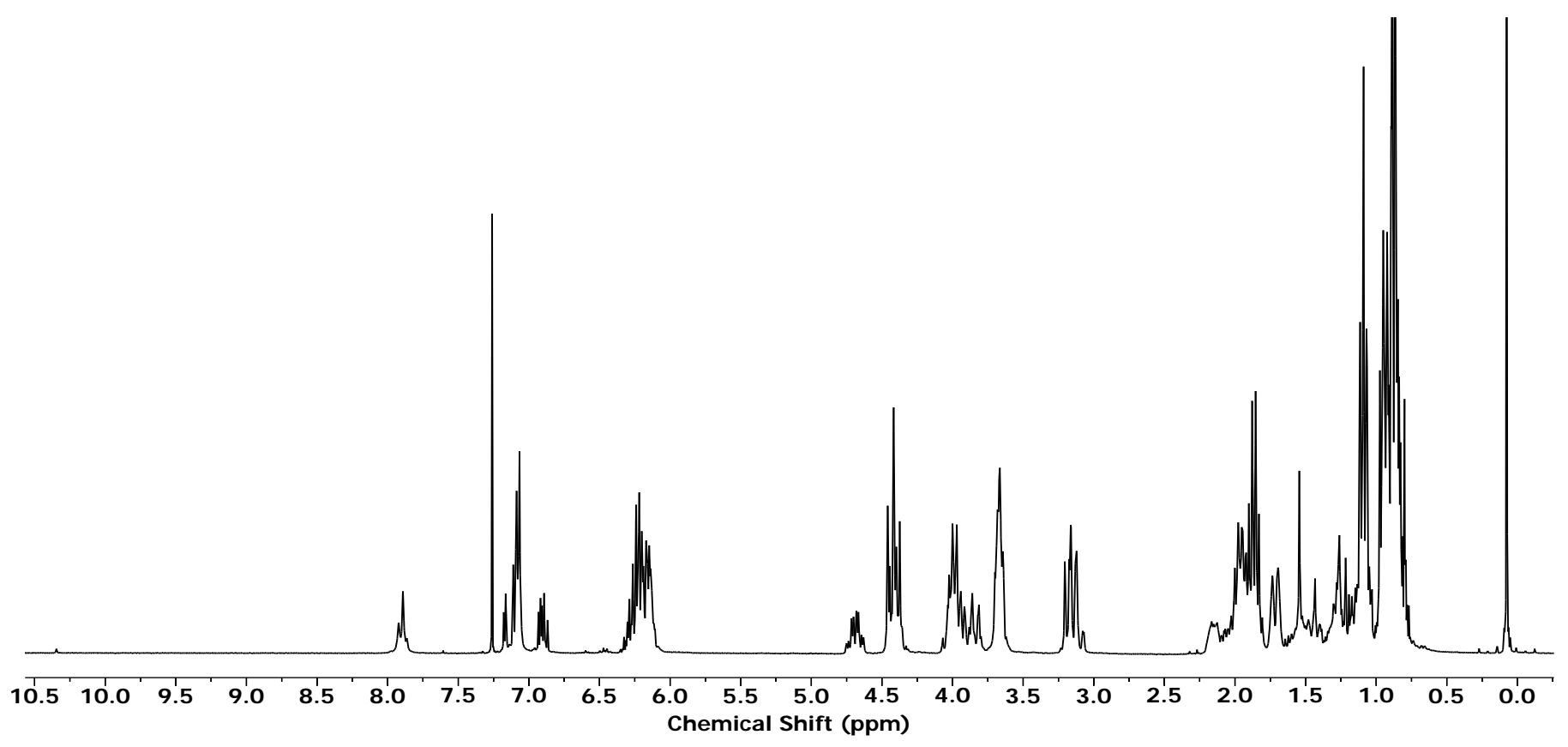

Figure 14. ${ }^{1} \mathrm{H}$ NMR spectrum (300 MHz, CDCl 3 ) for 4-bromo-5-menthyl carbamate-25,26,27,28-tetrapropoxycalix[4]arene 7a and 7b

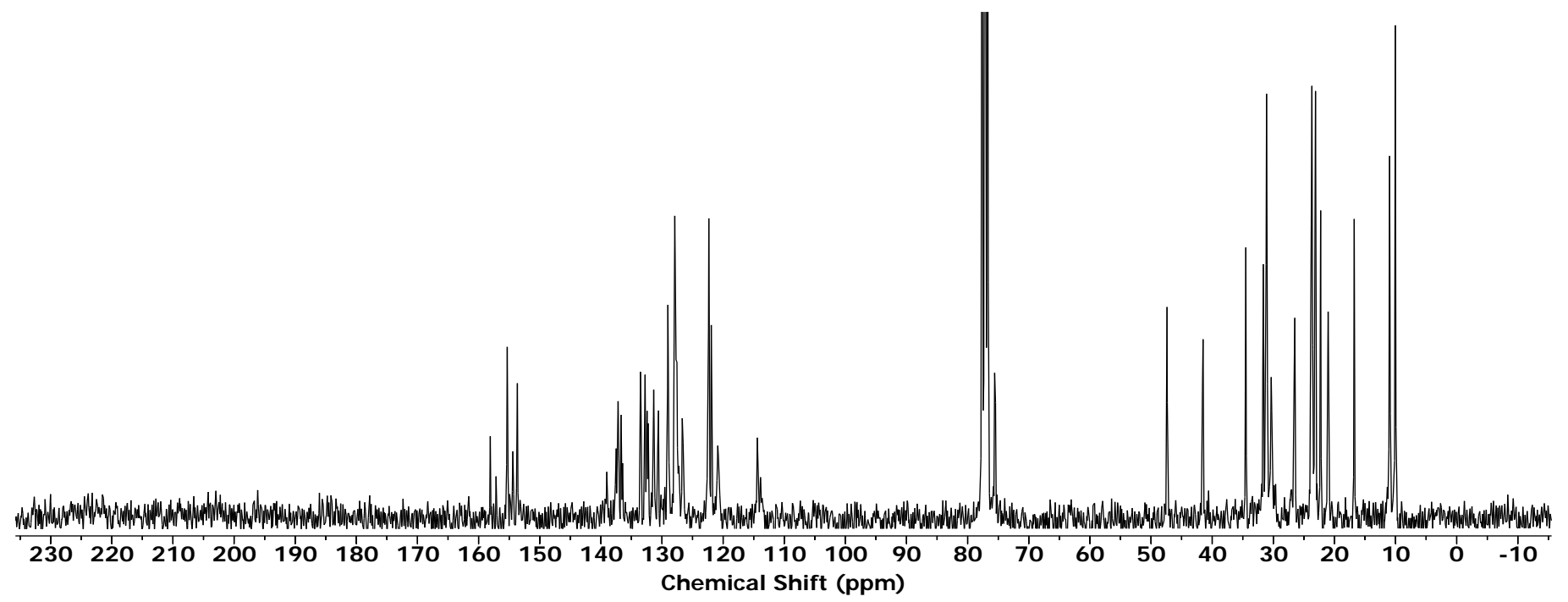

Figure 15. ${ }^{13} \mathrm{C} \mathrm{NMR} \mathrm{spectrum} \mathrm{(75} \mathrm{MHz,} \mathrm{CDCl} 3$ ) for 4-bromo-5-menthyl carbamate-25,26,27,28-tetrapropoxycalix[4]arene 7a and 7b 


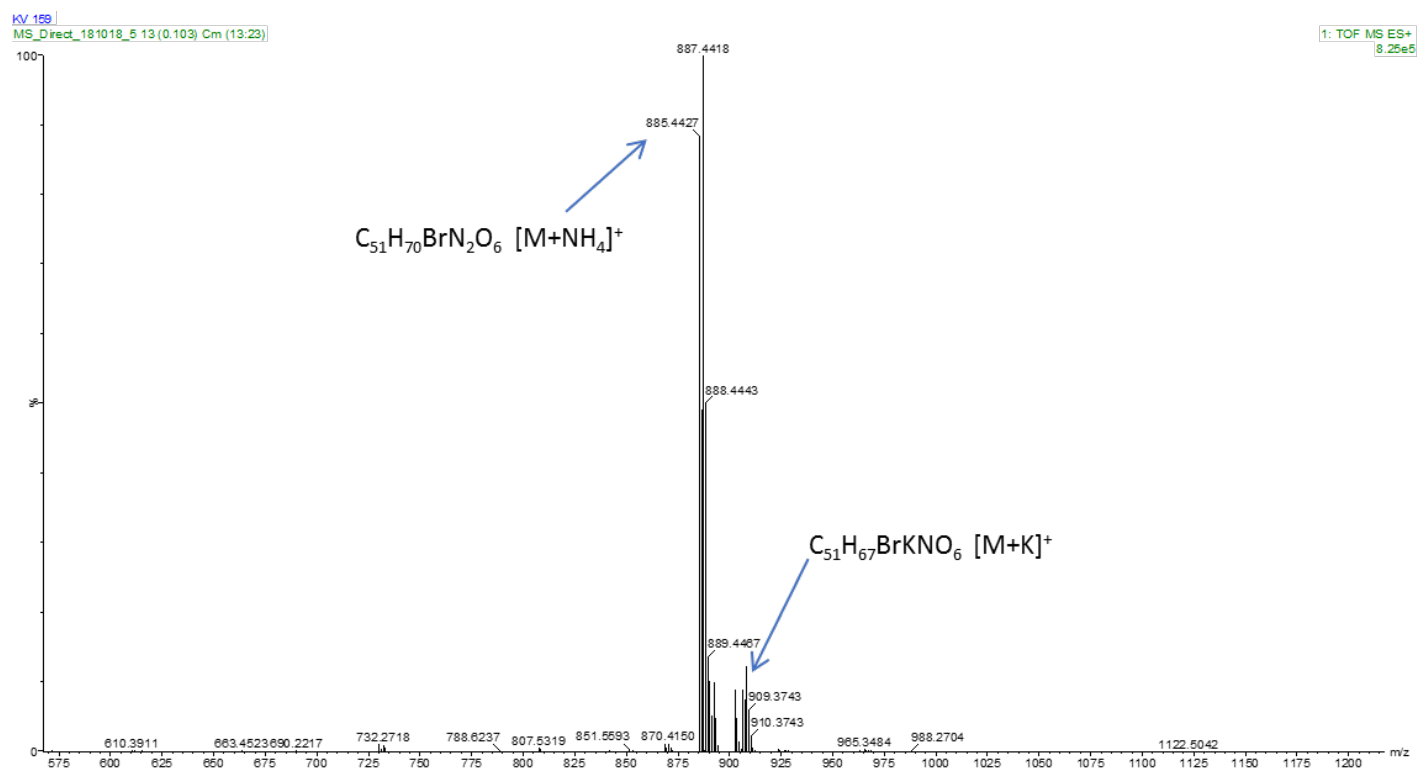

Figure 16. HRMS spectrum (ESI+) for 4-bromo-5-menthyl carbamate-25,26,27,28-tetrapropoxycalix[4]arene $7 \mathbf{a}$ and $7 \mathrm{~b}$

\section{4-Bromo-5-amino-25,26,27,28-tetrapropoxycalix[4]arene (8)}

IIVisagie $\mid C h a p t e r ~ 3$ - Mono Carbamate Calixarenes \(12)-4-bromo-5-amino-25,26,27,28-tetrapropoxy-calix[4]arene

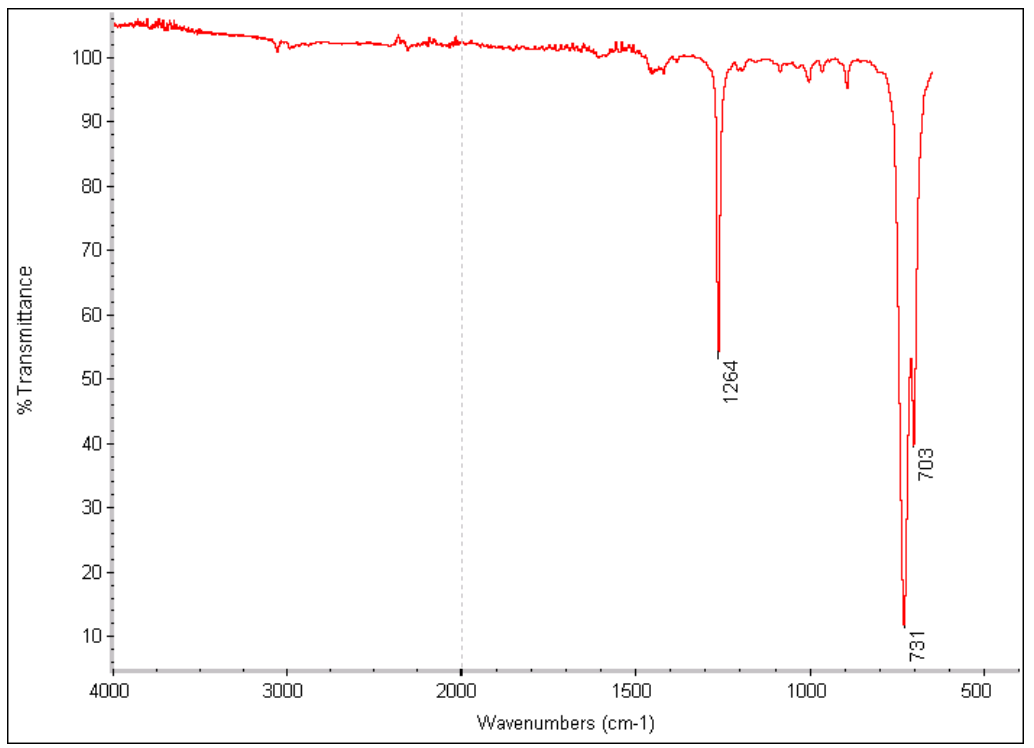

Figure 17. IR spectrum (ATR) for 4-bromo-5-amino-25,26,27,28-tetrapropoxycalix[4]arene $8 a$ and $8 b$ 


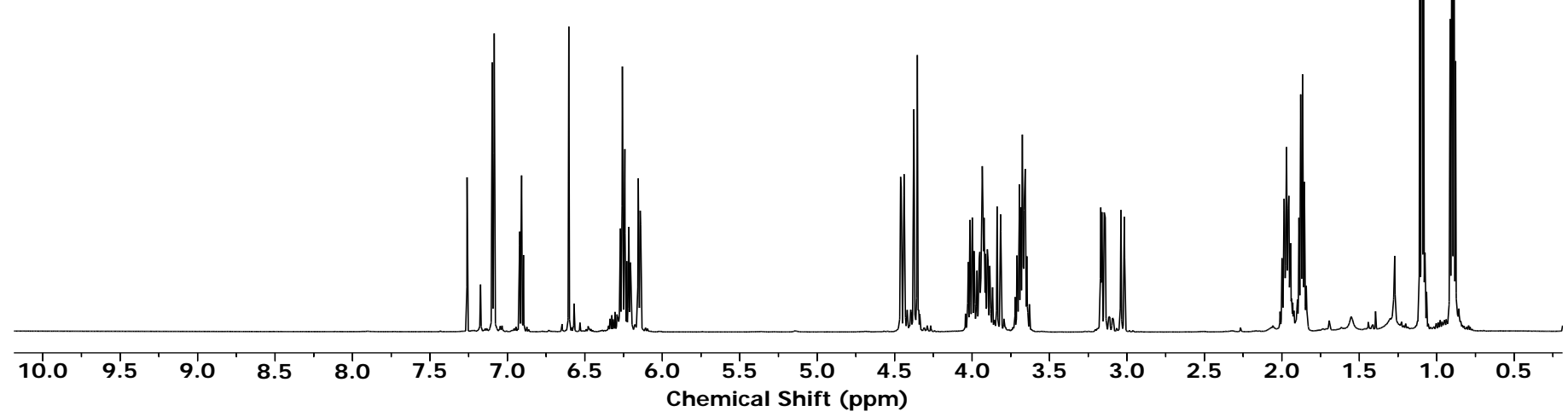

Figure 18. ${ }^{1} \mathrm{H}$ NMR spectrum (600 MHz, $\left.\mathrm{CDCl}_{3}\right)$ for 4-bromo-5-amino-25,26,27,28-tetrapropoxycalix[4]arene 8a and 8b

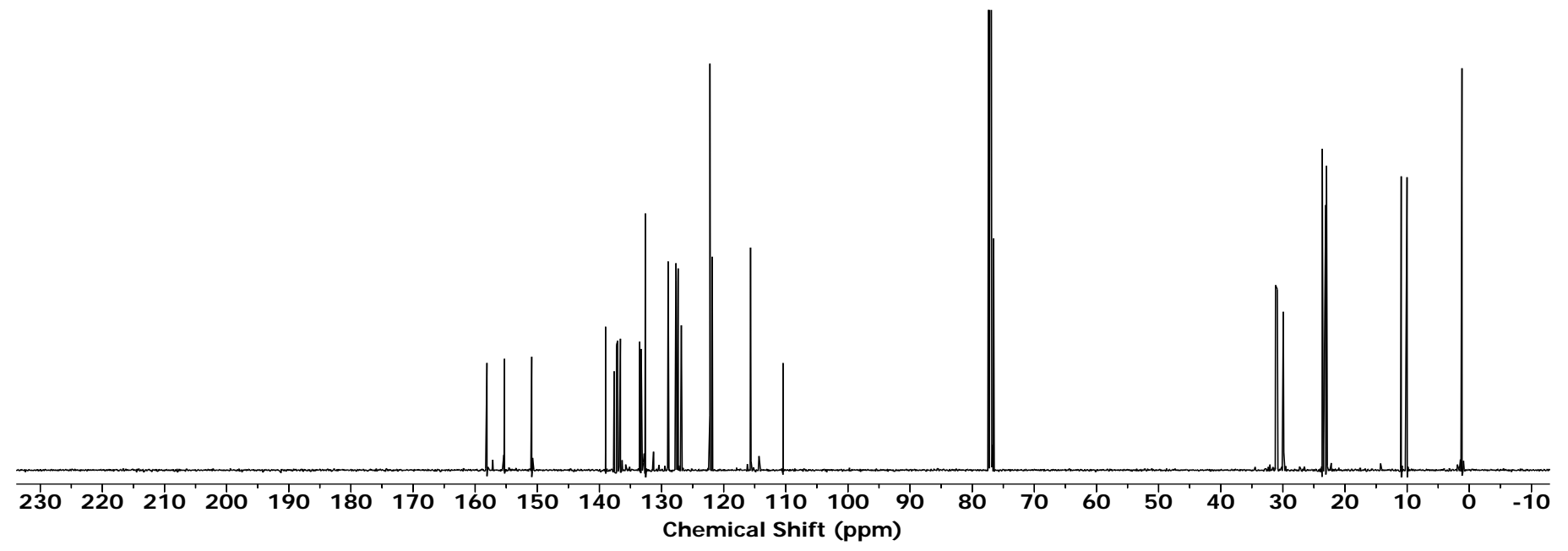

Figure 19. ${ }^{13} \mathrm{C} \mathrm{NMR} \mathrm{spectrum} \mathrm{(600} \mathrm{MHz,} \mathrm{CDCl}_{3}$ ) for 4-bromo-5-amino-25,26,27,28-tetrapropoxycalix[4]arene $8 a$ and $8 b$

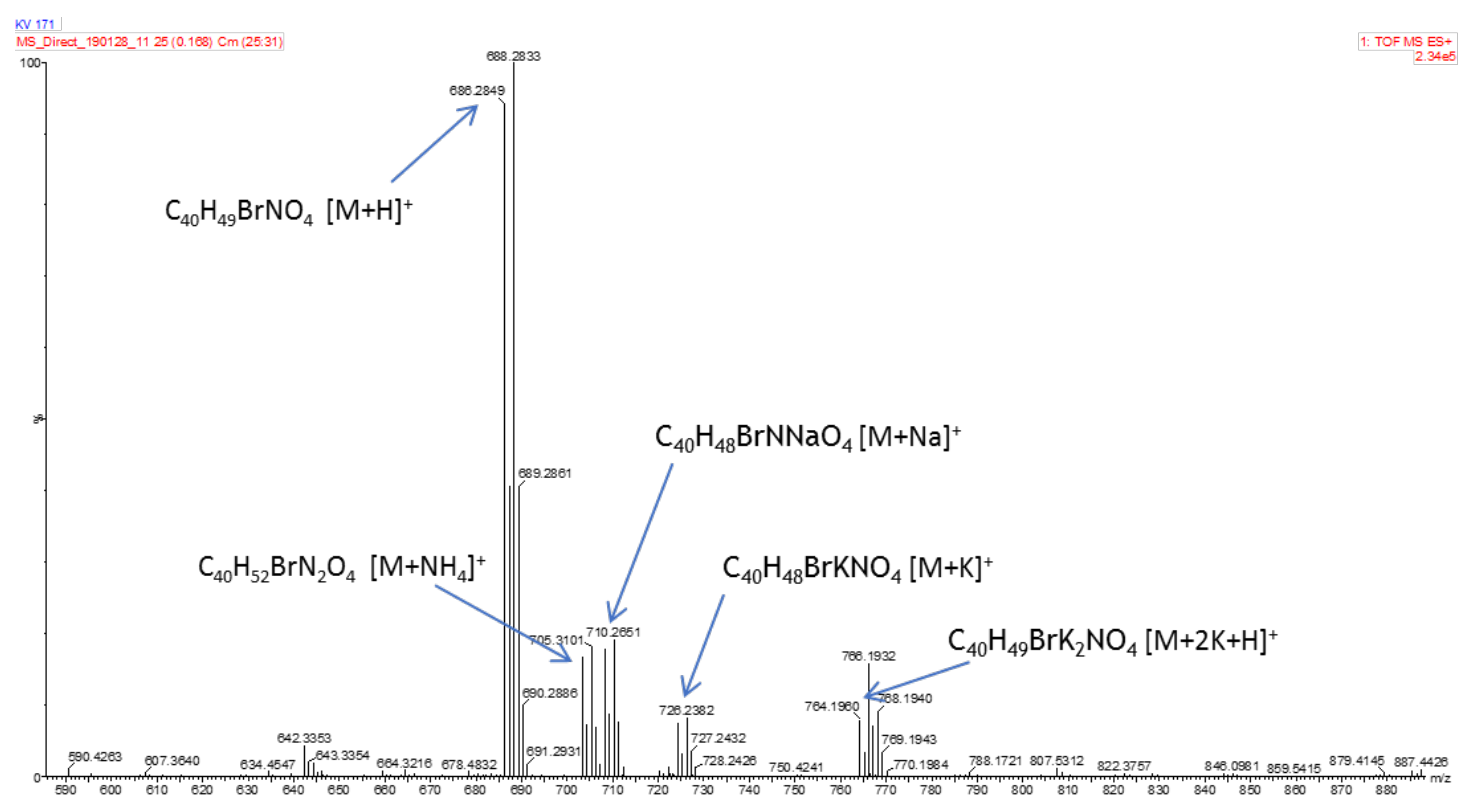

Figure 20. HRMS spectrum (ESI+) for 4-bromo-5-amino-25,26,27,28-tetrapropoxycalix[4]arene $8 a$ and $\mathbf{8 b}$ 
References for reactions where halogenation directed by a carbamate have been reported:

\section{Using NCS}

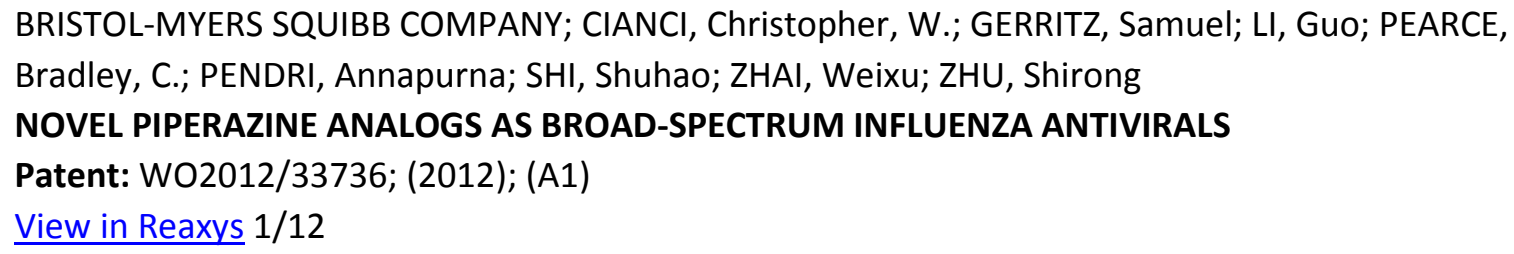


Richter Gedeon Vegyészeti Gyár RT.

\section{PROCESS FOR THE SYNTHESIS OF A BENZAMIDE DERIVATIVE}

Patent: WO2003/106440; (2003); (A2)

View in Reaxys $9 / 12$

Herrinton, Paul M.; Owen, Carolyn E.; Gage, James R.

lodination and metal halogen exchange of aromatic compounds: An improved preparation of a key oxazolidinone antibiotic intermediate

Organic Process Research and Development; vol. 5; nb. 1; (2001); p. 80 - 83

View in Reaxys 10/12

Xue Liang Tao; Cheng; Nishiyama; Yamamura

Synthetic studies on tetrahydropyrroloquinoline-containing natural products: Syntheses of discorhabdin $\mathbf{C}$, batzelline $\mathbf{C}$ and isobatzelline $\mathrm{C}$

Tetrahedron; vol. 50; nb. 7; (1994); p. 2017 - 2028

View in Reaxys $11 / 12$

Tao, Xue Liang; Nishiyama, Shigeru; Yamamura, Shosuke

Total Syntheses of Batzelline $C$ and Isobatzelline $C$, the Novel Pyrroloquinoline Alkaloids Isolated from the Marine Sponge Batzella Sp.

Chemistry Letters; nb. 10; (1991); p. 1785 - 1786

View in Reaxys 12/12

\section{Using NBS}

Chugai Seiyaku Kabushiki Kaisha; MURATA, Takeshi; KAWADA, Hatsuo; NIIZUMA, Satoshi; HARA, Sousuke; HADA, Kihito; SHIMADA, Hideaki; TANAKA, Hiroshi; MIO, Toshiyuki

QUINAZOLINEDIONE DERIVATIVE

Patent: EP2842946; (2015); (A1)

View in Reaxys $1 / 43$

Astellas Pharma Inc.; KANAYAMA, Takatoshi; KUBOTA, Hideki; MATSUMOTO, Shunichiro; SAITO, Tomoyuki; SHIMIZU, Takafumi; KATOH, Naoto; MATSUI, Shigeo

INDOLECARBOXAMIDE DERIVATIVE

Patent: EP2873660; (2015); (A1)

View in Reaxys $2 / 43$

Elgersma, Ronald C.; Coumans, Ruud G. E.; Huijbregts, Tijl; Menge, Wiro M. P. B.; Joosten, John A. F.; Spijker, Henri J.; De Groot, Franciscus M. H.; Van Der Lee, Miranda M. C.; Ubink, Ruud; Van Den Dobbelsteen, Diels J.; Egging, David F.; Dokter, Wim H. A.; Verheijden, Gijs F. M.; Lemmens, Jacques M.; Timmers, C. Marco; Beusker, Patrick H.

Design, synthesis, and evaluation of linker-duocarmycin payloads: Toward selection of HER2targeting antibody-drug conjugate SYD985

Molecular Pharmaceutics; vol. 12; nb. 6; (2015); p. 1813 - 1835

View in Reaxys $3 / 43$

Nowak, Monika; Malinowski, Zbigniew; Fornal, Emilia; Jóźwiak, Andrzej; Parfieniuk, Ewa; Gajek, Gabriela; Kontek, Renata 
Substituted benzoquinazolinones. Part 2: Synthesis of amino-, and sulfanyl-derivatives of benzo[f]and benzo[h]quinazolinones

Tetrahedron; vol. 71; nb. 50; (2015); p. 9463 - 9473

View in Reaxys $4 / 43$

SYNTHON BIOPHARMACEUTICALS B.V.; HUIJBRGTS, Tijl; ELGERSMA, Ronald Christiaan; BEUSKER, Patrick Henry; JOOSTEN, Johannes Albertus Frederikus; COUMANS, Rudy Gerardus Elisabeth; SPIJKER, Henri Johannes; MENGE, Wiro; DE GROOT, Franciscus Mariuns Hendrikus

IMPROVED PROCESS FOR MAKING DUOCARMYCIN PRODRUGS

Patent: WO2015/185142; (2015); (A1)

View in Reaxys $5 / 43$

Nguyen, Bichlien H.; Perkins, Robert J.; Smith, Jake A.; Moeller, Kevin D.

Solvolysis, Electrochemistry, and Development of Synthetic Building Blocks from Sawdust Journal of Organic Chemistry; vol. 80; nb. 24; (2015); p. 11953 - 11962

View in Reaxys 6/43

Patil, Pravin C.; Lee, Moses

An efficient synthesis of furano analogs of duocarmycin C1 and C2: Seco-isocyclopropylfurano[e]indoline-trimethoxyindole and seco- cyclopropylfurano[f]quinolinetrimethoxyindole

Tetrahedron Letters; vol. 55; nb. 21; (2014); p. 3283 - 3285

View in Reaxys $7 / 43$

El-Deeb, Ibrahim M.; Rose, Faith J.; Healy, Peter C.; Von Itzstein, Mark A versatile synthesis of "tafuramycin A": A potent anticancer and parasite attenuating agent Organic and Biomolecular Chemistry; vol. 12; nb. 24; (2014); p. 4260 - 4264

View in Reaxys 8/43

Zhang, Yu-Chen; Jiang, Fei; Wang, Shu-Liang; Shi, Feng; Tu, Shu-Jiang

Organocatalytic chemo- and regioselective oxyarylation of styrenes via a cascade reaction: Remote activation of hydroxyl groups

Journal of Organic Chemistry; vol. 79; nb. 13; (2014); p. 6143 - 6152

View in Reaxys 9/43

Rong, Zhouting; Li, Qingjiang; Lin, Wenhan; Jia, Yanxing

Reagent-free synthesis of 2,3,4-polysubstituted tetrahydroquinolines: Application to the formal synthesis of $( \pm)$-martinellic acid and martinelline

Tetrahedron Letters; vol. 54; nb. 33; (2013); p. 4432 - 4434

View in Reaxys 10/43

RVX Therapeutics Inc.; McLure, Kevin G.; Young, Peter Ronald

TREATMENT OF DISEASES BY EPIGENETIC REGULATION

Patent: US2013/281397; (2013); (A1)

View in Reaxys $11 / 43$

Guyonnet, Mathieu; Baudoin, Olivier

Synthesis of tricyclic nitrogen heterocycles by a sequence of palladium-catalyzed $\mathrm{N}-\mathrm{H}$ and $\mathrm{C}\left(\mathrm{sp}^{3}\right)-\mathrm{H}$ 


\section{arylations}

Organic Letters; vol. 14; nb. 1; (2012); p. 398 - 401

View in Reaxys 12/43

BRISTOL-MYERS SQUIBB COMPANY; CIANCI, Christopher, W.; GERRITZ, Samuel; LI, Guo; PEARCE, Bradley, C.; PENDRI, Annapurna; SHI, Shuhao; ZHAI, Weixu; ZHU, Shirong

NOVEL PIPERAZINE ANALOGS AS BROAD-SPECTRUM INFLUENZA ANTIVIRALS

Patent: WO2012/33736; (2012); (A1)

View in Reaxys $13 / 43$

Inoue, Keisuke; Ishikawa, Yuichi; Nishiyama, Shigeru

Synthesis of tetrahydropyrroloiminoquinone alkaloids based on electrochemically generated hypervalent iodine oxidative cyclization

Organic Letters; vol. 12; nb. 3; (2010); p. 436 - 439

View in Reaxys 14/43

Wilson, Kenneth J.; Illig, Carl R.; Chen, Jinsheng; Wall, Mark J.; Ballentine, Shelley K.; Desjarlais, Renee L.; Chen, Yanmin; Schubert, Carsten; Donatelli, Robert; Petrounia, loanna; Crysler, Carl S.; Molloy, Christopher J.; Chaikin, Margery A.; Manthey, Carl L.; Player, Mark R.; Tomczuk, Bruce E.; Meegalla, Sanath K.

Reducing ion channel activity in a series of 4-heterocyclic arylamide FMS inhibitors

Bioorganic and Medicinal Chemistry Letters; vol. 20; nb. 13; (2010); p. 3925 - 3929

View in Reaxys 15/43

RESVERLOGIX CORP.; HANSEN, Henrik, C.; WAGNER, Gregory, S.; ATTWELL, Sarah, C.; MCLURE, Kevin, G.; KULIKOWSKI, Ewelina, B.

NOVEL ANTI-INFLAMMATORY AGENTS

Patent: WO2010/123975; (2010); (A1)

View in Reaxys 16/43

Tietze, Lutz F.; Behrendt, Frank; Major, Felix; Krewer, Birgit; Von Hof, J. Marian

Synthesis of fluorescence-labelled glycosidic prodrugs based on the cytotoxic antibiotic duocarmycin

European Journal of Organic Chemistry; nb. 36; (2010); p. 6909 - 6921

View in Reaxys $17 / 43$

THE SCRIPPS RESEARCH INSTITUTE

CBI DERIVATIVES SUBJECT TO REDUCTIVE ACTIVATION

Patent: WO2009/64908; (2009); (A1)

View in Reaxys 18/43

MacMillan, Karen S.; Nguyen, Trihn; Hwang, Inkyu; Boger, Dale L.

Total synthesis and evaluation of iso-duocarmycin SA and iso-yatakemycin

Journal of the American Chemical Society; vol. 131; (2009); p. 1187 - 1194

View in Reaxys 19/43 
Patent: WO2009/144632; (2009); (A1)

View in Reaxys 20/43

Milbank, Jared B. J.; Stevenson, Ralph J.; Ware, David C.; Chang, John Y. C.; Tercel, Moana; Ahn, G.One; Wilson, William R.; Denny, William A.

Synthesis and evaluation of stable bidentate transition metal complexes of 1-(chloromethyl)-5hydroxy-3-(5,6,7-trimethoxyindol-2-ylcarbonyl)-2, 3-dihydro-1H-pyrrolo[3,2-f]quinoline (seco-6azaCBI-TMI) as hypoxia selective cytotoxins

Journal of Medicinal Chemistry; vol. 52; nb. 21; (2009); p. 6822 - 6834

View in Reaxys 21/43

Tercel, Moana; Atwell, Graham J.; Yang, Shangjin; Stevenson, Ralph J.; Botting, K. Jane; Boyd, Maruta; Smith, Eileen; Anderson, Robert F.; Denny, William A.; Wilson, William R.; Pruijn, Frederik B.

Hypoxia-activated prodrugs: Substituent effects on the properties of nitro seco-1,2,9,9atetrahydrocyclopropa[c]benz[e]indol-4-one (nitroCBI) prodrugs of DNA minor groove alkylating agents

Journal of Medicinal Chemistry; vol. 52; nb. 22; (2009); p. 7258 - 7272

View in Reaxys 22/43

Gauss, Carla M.; Hamasaki, Akiyuki; Parrish, Jay P.; MacMillan, Karen S.; Rayl, Thomas J.; Hwang, Inkyu; Boger, Dale L.

Synthesis and preliminary evaluation of duocarmycin analogues incorporating the 1,2,11,11atetrahydrocyclopropa[c]naphtho[2,3-e]indol-4-one (CNI) and 1,2,11,11a-

tetrahydrocyclopropa[c]naphtho[1,2-e]indol-4-one (iso-CNI) alkylation subunits

Tetrahedron; vol. 65; nb. 33; (2009); p. 6591 - 6599

View in Reaxys 23/43

Wong, Norman C.W.; Tucker, Joseph E.L.; Hansen, Henrik C.; Chiacchia, Fabrizio S.; McCaffrey, David COMPOUNDS FOR THE PREVENTION AND TREATMENT OF CARDIOVASCULAR DISEASES

Patent: US2008/188467; (2008); (A1)

View in Reaxys $24 / 43$

Illig, Carl R.; Ballentine, Shelley K.; Chen, Jinsheng; DesJarlais, Renee Louise; Meegalla, Sanath K.;

Wall, Mark; Wilson, Kenneth

INHIBITORS OF C-FMS KINASE

Patent: US2007/249593; (2007); (A1)

View in Reaxys 25/43

AUCKLAND UNISERVICES LIMITED

NITROBENZINDOLES AND THEIR USE IN CANCER THERAPY

Patent: WO2006/43839; (2006); (A1)

View in Reaxys 26/43

THE GOVERNMENT OF THE UNITED STATES OF AMERICA, AS REPRESENTED BY THE SECRETARY, DEPARTMENT OF HEALTH AND HUMAN SERVICES

BUILDING BLOCKS FOR DNA BINDING AGENTS

Patent: WO2005/32594; (2005); (A2)

View in Reaxys 27/43 
Ennis, Michael D.; Frank, Kristine E.; Hoffman, Robert L.; Fu, Jian-Min

THERAPEUTIC 1H-PYRIDO [ 4, 3-B ] INDOLES

Patent: US2003/60464; (2003); (A1)

View in Reaxys 28/43

Tietze, Lutz F.; Haunert, Frank; Feuerstein, Tim; Herzig, Tobias

A concise and efficient synthesis of seco-duocarmycin SA

European Journal of Organic Chemistry; nb. 3; (2003); p. 562 - 566

View in Reaxys 29/43

Chassot, Laurent; Braun, Hans-Juergen

Dyes for keratin fibres containing 1,3-diamino-4-heteroarylbenzene derivatives and novel 1,3-

diamino-4-heteroarylbenzene derivatives

Patent: US2003/93867; (2003); (A1)

View in Reaxys 30/43

Howard, Tiffany T; Lingerfelt, Brian M; Purnell, Bethany L; Scott, Adrienne E; Price, Carly A; Townes, Heather M; McNulty, LuAnne; Handl, Heather L; Summerville, Kaitlin; Hudson, Stephen J; Bowen, J Phillip; Kiakos, Konstantinos; Hartley, John A; Lee, Moses

Novel furano analogues of duocarmycin C1 and C2: design, synthesis, and biological evaluation of seco-iso-cyclopropylfurano[2,3-e]indoline (seco-iso-CFI) and seco-cyclopropyltetrahydrofurano[2,3f]quinoline (seco-CFQ) analogues.

Bioorganic and medicinal chemistry; vol. 10; nb. 9; (2002); p. 2941 - 2952

View in Reaxys 31/43

Boger; Hughes; Hedrick

Synthesis, chemical properties, and biological evaluation of CC-1065 and duocarmycin analogues incorporating the 5-methoxycarbonyl-1,2,9,9a-tetrahydrocyclopropa[c]benz[e]indol-4-one alkylation subunit

Journal of Organic Chemistry; vol. 66; nb. 7; (2001); p. 2207 - 2216

View in Reaxys 32/43

The Scripps Research Institute

Analogs of CC-1065 and the duocarmycins

Patent: US6060608; (2000); (A)

View in Reaxys 33/43

The Scripps Research Institute

$\mathrm{MCBI}$ analogs of CC-1065 and the duocarmycins

Patent: US5985908; (1999); (A)

View in Reaxys $34 / 43$

Patel, Vinod F.; Andis, Sherri L.; Enkema, Julia K.; Johnson, David A.; Kennedy, Joseph H.; Mohamadi, Fariborz; Schultz, Richard M.; Soose, Daniel J.; Spees, Michael M.

Total synthesis of seco (+)- and ent-(-)-oxaduocarmycin SA: Construction of the (chloromethyl)indoline alkylating subunit by a novel intramolecular aryl radical cyclization onto a vinyl chloride

Journal of Organic Chemistry; vol. 62; nb. 25; (1997); p. 8868 - 8874 
Boger, Dale L.; McKie, Jeffrey A.; Cai, Hui; Cacciari, Barbara; Baraldi

Synthesis and properties of substituted CBI analogs of CC-1065 and the duocarmycins incorporating the 7-methoxy-1,2,9,9a-tetrahydrocyclopropa[c]benz[e]indol-4-one (MCBI) alkylation subunit: Magnitude of electronic effects on the functional reactivity Journal of Organic Chemistry; vol. 61; nb. 5; (1996); p. 1710 - 1729 View in Reaxys 36/43

Boger, Dale L.; Han, Nianhe; Tarby, Christine M.; Boyce, Christopher W.; Cai, Hui; Jin, Qing; Kitos, Paul A.

Synthesis, chemical properties, and preliminary evaluation of substituted CBI analogs of CC-1065 and the duocarmycins incorporating the 7-cyano-1,2,9,9a-tetrahydrocyclopropa[c]benz[e]indol-4one alkylation subunit: Hammett quantitation of the magnitude of electronic effects on functional reactivity

Journal of Organic Chemistry; vol. 61; nb. 15; (1996); p. 4894 - 4912

View in Reaxys $37 / 43$

Mohamadi, Fariborz; Spees, Michael M.; Staten, Gilbert S.; Marder, Philip; Kipka, Julia K.; et al.

Total Synthesis and Biological Properties of Novel Antineoplastic (Chloromethyl)furanoindolines: An Asymmetric Hydroboration Mediated Synthesis of the Alkylation Subunits Journal of Medicinal Chemistry; vol. 37; nb. 2; (1994); p. 232 - 239

View in Reaxys 38/43

Boger; Nishi; Teegarden

p-Quinomethide analog of the CC-1065 and duocarmycin alkylation subunits

Journal of Organic Chemistry; vol. 59; nb. 17; (1994); p. 4943 - 4949

View in Reaxys 39/43

Eli Lilly and Company

Furanoindolines

Patent: US5248691; (1993); (A)

View in Reaxys $40 / 43$

Boger, Dale L.; Yun, Weiya; Teegarden, Bradley R.

An Improved Synthesis of 1,2,9,9a-Tetrahydrocyclopropa<c>benz<e>indol-4-one (CBI): A Simplified Analogue of the CC-1065 Alkylation Subunit

Journal of Organic Chemistry; vol. 57; nb. 10; (1992); p. 2873 - 2876

View in Reaxys $41 / 43$

Boger, Dale L.; Ishizaki, Takayoshi; Kitos, Paul A.; Suntornwat, Oranart

Synthesis of N-(tert-Butyloxycarbonyl)-CBI, CBI, CBI-CDPI ${ }_{1}$, and CBI-CDPI ${ }_{2}$ : Enhanced Functional Analogues of CC-1065 Incorporating the 1,2,9,9a-Tetrahydrocyclopropa $<$ c $>$ benz<e $>$ indol-4-one (CBI) Left-Hand Subunit

Journal of Organic Chemistry; vol. 55; nb. 23; (1990); p. 5823 - 5832

View in Reaxys $42 / 43$

Boger, Dale L.; Ishizaki, Takayoshi; Wysocki, Ronald J.; Munk, Stephen A.; Kitos, Paul A.; Suntornwat, 


\section{Oranart}

Total Synthesis and Evaluation of (+/-)-N-(tert-Butyloxycarbonyl)-CBI, (+/-)-CBI-CDPI1, and (+/-)-

CBI-CDPI2: CC-1065 Functional Agents Incorporating the Equivalent 1,2,9,9a-

Tetrahydrocycloprop<1,2-c>benz<1,2-e>indol-4-one (CBI) Left-Hand Subunit

Journal of the American Chemical Society; vol. 111; nb. 16; (1989); p. 6461 - 6463

View in Reaxys $43 / 43$

\section{Using NIS}

Fra, Laura; Millán, Alba; Souto, José A.; Muñiz, Kilian Indole synthesis based on a modified koser reagent Angewandte Chemie - International Edition; vol. 53; nb. 28; (2014); p. 7349 - 7353

View in Reaxys $1 / 24$

Uematsu, Mika; Boger, Dale L.

Asymmetric synthesis of a CBI-based cyclic $\mathrm{N}$-acyl O-amino phenol duocarmycin prodrug Journal of Organic Chemistry; vol. 79; nb. 20; (2014); p. 9699 - 9703

View in Reaxys 2/24

THE SCRIPPS RESEARCH INSTITUTE; BOGER, Dale L.

CYCLIC PRODRUGS OF DUOCARMYCIN ANALOGS

Patent: WO2013/148631; (2013); (A1)

View in Reaxys 3/24

Zhao, Robert Yongxin; Erickson, Hans K.; Leece, Barbara A.; Reid, Emily E.; Goldmacher, Victor S.; Lambert, John M.; Chari, Ravi V. J.

Synthesis and biological evaluation of antibody conjugates of phosphate prodrugs of cytotoxic DNA alkylators for the targeted treatment of cancer

Journal of Medicinal Chemistry; vol. 55; nb. 2; (2012); p. 766 - 782

View in Reaxys 4/24

Stevenson, Ralph J.; Denny, William A.; Tercel, Moana; Pruijn, Frederik B.; Ashoorzadeh, Amir Nitro seco analogues of the duocarmycins containing sulfonate leaving groups as hypoxia-activated prodrugs for cancer therapy

Journal of Medicinal Chemistry; vol. 55; nb. 6; (2012); p. 2780 - 2802

View in Reaxys 5/24

Wolfe, Amanda L.; Duncan, Katharine K.; Parelkar, Nikhil K.; Weir, Scott J.; Vielhauer, George A.; Boger, Dale L.

A novel, unusually efficacious duocarmycin carbamate prodrug that releases no residual byproduct Journal of Medicinal Chemistry; vol. 55; nb. 12; (2012); p. 5878 - 5886

View in Reaxys 6/24

Boyle, Kristopher E.; MacMillan, Karen S.; Ellis, David A.; Lajiness, James P.; Robertson, William M.;

Boger, Dale L.

Synthesis and evaluation of duocarmycin SA analogs incorporating the methyl $1,2,8,8 \mathrm{a}-$ tetrahydrocyclopropa[c]oxazolo[2,3-e]indol-4-one-6-carboxylate (COI) alkylation subunit Bioorganic and Medicinal Chemistry Letters; vol. 20; nb. 6; (2010); p. 1854 - 1857 


\section{F. Hoffmann-La Roche AG}

Process for the preparation of an indole derivative

Patent: EP2011783; (2009); (A1)

View in Reaxys $8 / 24$

Milbank, Jared B. J.; Stevenson, Ralph J.; Ware, David C.; Chang, John Y. C.; Tercel, Moana; Ahn, G.One; Wilson, William R.; Denny, William A.

Synthesis and evaluation of stable bidentate transition metal complexes of 1-(chloromethyl)-5hydroxy-3-(5,6,7-trimethoxyindol-2-ylcarbonyl)-2, 3-dihydro-1H-pyrrolo[3,2-f]quinoline (seco-6azaCBI-TMI) as hypoxia selective cytotoxins

Journal of Medicinal Chemistry; vol. 52; nb. 21; (2009); p. 6822 - 6834

View in Reaxys 9/24

MacMillan, Karen S.; Lajiness, James P.; Cara, Carlota Lopez; Romagnoli, Romeo; Robertson, William M.; Hwang, Inkyu; Baraldi, Pier Giovanni; Boger, Dale L.

Synthesis and evaluation of a thio analogue of duocarmycin SA

Bioorganic and Medicinal Chemistry Letters; vol. 19; nb. 24; (2009); p. 6962 - 6965

View in Reaxys 10/24

Tietze, Lutz F.; Panknin, Olaf; Major, Felix; Krewer, Birgit

Synthesis of a novel pentagastrin-drug conjugate for a targeted tumor therapy

Chemistry - A European Journal; vol. 14; nb. 9; (2008); p. 2811 - 2818

View in Reaxys $11 / 24$

Faeh, Christoph; Kuehne, Holger; Luebbers, Thomas; Mattei, Patrizio; Maugeais, Cyrille; Pflieger, Philippe

Heteroaryl and benzyl amide compounds

Patent: US2007/185113; (2007); (A1)

View in Reaxys $12 / 24$

Conte, Aurelia; Kuehne, Holger; Luebbers, Thomas; Mattei, Patrizio; Maugeais, Cyrille; Mueller, Werner; Pflieger, Philippe

Heteroaryl carboxamide compounds

Patent: US2007/185182; (2007); (A1)

View in Reaxys 13/24

Tichenor, Mark S.; MacMillan, Karen S.; Stover, James S.; Wolkenberg, Scott E.; Pavani, Maria G.; Zanella, Lorenzo; Zaid, Abdel N.; Spalluto, Gianpiero; Rayl, Thomas J.; Hwang, Inkyu; Baraldi, Pier Giovanni; Boger, Dale L.

Rational design, synthesis, and evaluation of key analogues of CC-1065 and the duocarmycins Journal of the American Chemical Society; vol. 129; nb. 45; (2007); p. 14092 - 14099

View in Reaxys 14/24

MEDAREX, INC.

METHODS AND COMPOUNDS FOR PREPARING CC-1065 ANALOGS

Patent: WO2007/51081; (2007); (A1) 
Tichenor, Mark S.; Trzupek, John D.; Kastrinsky, David B.; Shiga, Futoshi; Hwang, Inkyu; Boger, Dale L. Asymmetric total synthesis of (+)- and ent-(-)-yatakemycin and duocarmycin SA: Evaluation of yatakemycin key partial structures and its unnatural enantiomer Journal of the American Chemical Society; vol. 128; nb. 49; (2006); p. 15683 - 15696

View in Reaxys 16/24

Conte-Mayweg, Aurelia; Kuehne, Holger; Luebbers, Thomas; Maugeais, Cyrille; Mueller, Werner; Pflieger, Philippe Indole, indazole and indoline derivatives as CETP inhibitors

Patent: US2006/30613; (2006); (A1)

View in Reaxys $17 / 24$

\section{Spirogen Limited}

Cyclopropylindole derivatives

Patent: US6909006; (2005); (B1)

View in Reaxys 18/24

Adams, David; Benardeau, Agnes; Bickerdike, Mike J.; Bentley, Jon M.; Bissantz, Caterina; Bourson, Anne; Cliffe, Ian A.; Hebeisen, Paul; Kennett, Guy A.; Knight, Antony R.; Malcolm, Craig S.; Mizrahi, Jacques; Plancher, Jean-Marc; Richter, Hans; Roever, Stephan; Taylor, Sven; Vickers, Steven P.

$5-\mathrm{HT}_{2 \mathrm{C}}$ receptor agonists for the treatment of obesity. Biological and chemical adventures

Chimia; vol. 58; nb. 9; (2004); p. 613 - 620

View in Reaxys 19/24

Bentley, Jonathan Mark; Bickerdike, Michael John; Hebeisen, Paul; Kennett, Guy Anthony; Lightowler, Sean; Mattei, Patrizio; Mizrahi, Jacques; Morley, Timothy James; Plancher, Jean-Marc; Richter, Hans; Roever, Stephan; Taylor, Sven; Vickers, Steven Paul

Novel indole derivatives

Patent: US2002/160997; (2002); (A1)

View in Reaxys 20/24

Boger; Brunette; Garbaccio

Synthesis and evaluation of a series of C3-substituted CBI analogues of CC-1065 and the duocarmycins

Journal of Organic Chemistry; vol. 66; nb. 15; (2001); p. 5163 - 5173

View in Reaxys 21/24

Boger; Boyce

Selective metal cation activation of a DNA alkylating agent: Synthesis and evaluation of methyl 1,2,9,9a-tetrahydrocyclopropa[c]pyrido[3,2-e]indol-4-one-7-carboxylate (CPyl)

Journal of Organic Chemistry; vol. 65; nb. 13; (2000); p. 4088 - 4100

View in Reaxys 22/24

Boger, Dale L.; McKie, Jeffrey A.; Cai, Hui; Cacciari, Barbara; Baraldi

Synthesis and properties of substituted CBI analogs of CC-1065 and the duocarmycins incorporating the 7-methoxy-1,2,9,9a-tetrahydrocyclopropa[c]benz[e]indol-4-one (MCBI) alkylation subunit: 


\section{Magnitude of electronic effects on the functional reactivity}

Journal of Organic Chemistry; vol. 61; nb. 5; (1996); p. 1710 - 1729

View in Reaxys 23/24

Boger, Dale L.; Han, Nianhe; Tarby, Christine M.; Boyce, Christopher W.; Cai, Hui; Jin, Qing; Kitos, Paul A.

Synthesis, chemical properties, and preliminary evaluation of substituted CBI analogs of CC-1065 and the duocarmycins incorporating the 7-cyano-1,2,9,9a-tetrahydrocyclopropa[c]benz[e]indol-4one alkylation subunit: Hammett quantitation of the magnitude of electronic effects on functional reactivity

Journal of Organic Chemistry; vol. 61; nb. 15; (1996); p. 4894 - 4912

View in Reaxys 24/24

\section{Using $\mathrm{Cl}_{2}$}

Hansen, John Bondo; Fink-Jensen, Anders; Christensen, Birgitte V.; Gronvald, Frederick C.; Jeppesen, Lone; Mogensen, John P.; Nielsen, Erik B.; Scheideler, Mark A.; White, Francis J.; Zhang, Xu-Feng Mesolimbic selective antipsychotic arylcarbamates

European Journal of Medicinal Chemistry; vol. 33; nb. 11; (1998); p. 839 - 858

View in Reaxys $1 / 7$

Yamanouchi Pharmaceutical Co., Ltd.

4-SUBSTITUTED AMINO-ALPHA-AMINOMETHYLBENZYL ALCOHOL DERIVATIVES

Patent: US4063025; (1977); (A)

View in Reaxys $2 / 7$

Yamanouchi Pharm.

Patent: FR2299855; (1976)

DE2603417; (1976)

Chem.Abstr.; vol. 85; nb. 159632; (1976)

View in Reaxys $3 / 7$

Pan; Fletcher

Derivatives of fluorene. XXI. New halogenofluorenes. II. Further potential antitumor agents.

Journal of medicinal chemistry; vol. 8; nb. 4; (1965); p. 491 - 497

View in Reaxys 4/7

Cosulich et al.

Journal of the American Chemical Society; vol. 75; (1953); p. 4675,4680

View in Reaxys $5 / 7$

Matheson; McCombie

Journal of the Chemical Society; (1931); p. 1103,1108

View in Reaxys 6/7

Buchan; McCombie

Journal of the Chemical Society; (1931); p. 137,144 


\section{Using $\mathrm{Br}_{2}$}

Isley, Nicholas A.; Dobarco, Sebastian; Lipshutz, Bruce H.

Installation of protected ammonia equivalents onto aromatic \& heteroaromatic rings in water enabled by micellar catalysis

Green Chemistry; vol. 16; nb. 3; (2014); p. 1480 - 1488

View in Reaxys $1 / 18$

Hashimoto, Takuya; Nakatsu, Hiroki; Takiguchi, Yuka; Maruoka, Keiji

Axially chiral dicarboxylic acid catalyzed activation of quinone imine ketals: Enantioselective arylation of enecarbamates

Journal of the American Chemical Society; vol. 135; nb. 43; (2013); p. 16010 - 16013

View in Reaxys 2/18

Evans, Kathryn M.; Haraldsen, Jeralyn D.; Pearson, Russell J.; Slawin, Alexandra M. Z.; Ward, Gary E.; Westwood, Nicholas J.

Synthesis and chemical characterisation of target identification reagents based on an inhibitor of human cell invasion by the parasite Toxoplasma gondii

Organic and Biomolecular Chemistry; vol. 5; nb. 13; (2007); p. 2063 - 2069

View in Reaxys $3 / 18$

LANXESS Deutschland GmbH

Process for the preparation of carbamic acid derivatives

Patent: EP1669346; (2006); (A1)

View in Reaxys $4 / 18$

Ishida, Junya; Yamamoto, Hirofumi; Kido, Yoshiyuki; Kamijo, Kazunori; Murano, Kenji; Miyake, Hiroshi; Ohkubo, Mitsuru; Kinoshita, Takayoshi; Warizaya, Masaichi; Iwashita, Akinori; Mihara, Kayoko; Matsuoka, Nobuya; Hattori, Kouji

Discovery of potent and selective PARP-1 and PARP-2 inhibitors: SBDD analysis via a combination of X-ray structural study and homology modeling

Bioorganic and Medicinal Chemistry; vol. 14; nb. 5; (2006); p. 1378 - 1390

View in Reaxys $5 / 18$

Ishida, Junya; Hattori, Kouji; Yamamoto, Hirofumi; Iwashita, Akinori; Mihara, Kayoko; Matsuoka, Nobuya

4-Phenyl-1,2,3,6-tetrahydropyridine, an excellent fragment to improve the potency of PARP-1 inhibitors

Bioorganic and Medicinal Chemistry Letters; vol. 15; nb. 19; (2005); p. 4221 - 4225

View in Reaxys 6/18

Frank, Kristine E.; Aube, Jeffrey

Cyclizations of substituted benzylidene-3-alkenylamines: Synthesis of the tricyclic core of the martinellines

Journal of Organic Chemistry; vol. 65; nb. 3; (2000); p. 655 - 666 
Hansen, John Bondo; Fink-Jensen, Anders; Christensen, Birgitte V.; Gronvald, Frederick C.; Jeppesen, Lone; Mogensen, John P.; Nielsen, Erik B.; Scheideler, Mark A.; White, Francis J.; Zhang, Xu-Feng Mesolimbic selective antipsychotic arylcarbamates European Journal of Medicinal Chemistry; vol. 33; nb. 11; (1998); p. $839-858$ View in Reaxys $8 / 18$

Nikam, Sham S.; Yuen, Po-Wai; Kornberg, Brian E.; Tobias, Brian; Rafferty, Michael F. Novel Use of Substituted 1,4-Dihydrobenz $<d><1,3>0 x a z i n-2-o n e s$ in the Synthesis of Important Aminomethyl o-Nitroanilines Journal of Organic Chemistry; vol. <26> 62; (1997); p. 9331 - 9334

View in Reaxys $9 / 18$

Novo Nordisk A/S

ANTIPSYCHOTIC PIPERIDINE DERIVATIVES

Patent: US5378714; (1995); (A)

View in Reaxys $10 / 18$

Cambie, Richard C.; Higgs, Paul I.; Rutledge, Peter S.; Woodgate, Paul D.

Aryne Chemistry of Podocarpic Acid Derivatives

Australian Journal of Chemistry; vol. 47; nb. 8; (1994); p. 1483 - 1508

View in Reaxys $11 / 18$

Bengtsson, Stefan; Hoegberg, Thomas

Secondary $\beta$-Aminobenzamide and Heteroatom Directed Lithiation in the Synthesis of 5,6-

Dimethoxyanthanilamides and Related Compounds

Journal of Organic Chemistry; vol. 54; nb. 19; (1989); p. 4549 - 4553

View in Reaxys 12/18

Schmidt, Hans-Werner

Synthesis of new 4-Hydroxybenzonitrile Derivatives and their Herbicidal Properties

Monatshefte fuer Chemie; vol. 118; (1987); p. 217 - 228

View in Reaxys 13/18

Ishii, Hisashi; Murakami, Yasuoki; Watanabe, Toshiko; Suzuki, Hideharu; Maejima, Hideyuki Fischer Indolization and Its Related Compounds. XIX. Synthesis of Ethyl 4-Methoxy- and Ethyl 5Methoxy-1-phenyl-3H-benz<e>indole-2-carboxylates

Chemical \& Pharmaceutical Bulletin; vol. 31; nb. 12; (1983); p. 4401 - 4408

View in Reaxys 14/18

Yamanouchi Pharm.

Patent: FR2299855; (1976)

DE2603417; (1976)

Chem.Abstr.; vol. 85; nb. 159632; (1976)

View in Reaxys 15/18 
Journal of the American Chemical Society; vol. 81; (1959); p. 4983,4986

View in Reaxys 16/18

Lesslie; Turner

Journal of the Chemical Society; (1933); p. 1588,1591

View in Reaxys $17 / 18$

I.G.Farbenind.

Patent: DE621710; (1933)

Fortschr. Teerfarbenfabr. Verw. Industriezweige; vol. 21; p. 299

View in Reaxys 18/18

\section{Using $I_{2}$}

Tietze, Lutz F.; Herzig, Tobias; Feuerstein, Tim; Schuberth, Ingrid

Synthesis and biological evaluation of novel analogues and prodrugs of the cytotoxic antibiotic CC1065 for selective cancer therapy

European Journal of Organic Chemistry; nb. 10; (2002); p. 1634 - 1645

View in Reaxys $1 / 3$

Gallo-Rodriguez, Carola; Ji, Xiao-duo; Melman, Neli; Siegman, Barry D.; Sanders, Lawrence H.; et al. Structure-Activity Relationships of $\mathbf{N}^{6}$-Benzyladenosine-5'-uronamides as $\mathbf{A}_{3}$-Selective Adenosine Agonists

Journal of Medicinal Chemistry; vol. 37; nb. 5; (1994); p. 636 - 646

View in Reaxys $2 / 3$

Schmidt, Hans-Werner

Synthesis of new 4-Hydroxybenzonitrile Derivatives and their Herbicidal Properties

Monatshefte fuer Chemie; vol. 118; (1987); p. 217 - 228

View in Reaxys $3 / 3$ 